(c) 2004 International Press

Adv. Theor. Math. Phys. 8 (2004) 345-394

\title{
M-theory, type IIA superstrings, and elliptic cohomology
}

\author{
Igor Kriz \\ ikriz@umich.edu . \\ Department of Mathematics \\ University of Michigan \\ Ann Arbor, MI 48109, \\ USA
}

\author{
Hisham Sati \\ hsati@maths.adelaide.edu.au. \\ Department of Physics \\ University of Adelaide \\ Adelaide, SA 5005, \\ Australia \\ Department of Pure Mathematics \\ University of Adelaide \\ Adelaide, SA 5005, \\ Australia
}

\begin{abstract}
The topological part of the M-theory partition function was shown by Witten to be encoded in the index of an $E_{8}$ bundle in eleven dimensions. This partition function is, however, not automatically anomalyfree. We observe here that the vanishing $W_{7}=0$ of the DiaconescuMoore-Witten anomaly [1] in IIA and compactified M-theory partition function is equivalent to orientability of spacetime with respect to (complex-oriented) elliptic cohomology. Motivated by this, we define an elliptic cohomology correction to the IIA partition function, and propose its relationship to interaction between 2- and 5-branes in the M-theory limit.
\end{abstract}

e-print archive: http://lanl.arXiv.org/abs/hep-th/0404013 


\section{Introduction}

There are two superstring theories with two supersymmetries in ten dimensions. One is nonchiral $N=(1,1)$ type IIA string theory and the other is chiral $N=(2,0)$ IIB theory. Their fields fall into two sectors, the RR (Ramond-Ramond) sector and the NS (Neveu-Schwarz) sector. The RR fields naturally couple to D-branes [2] of even (odd) spatial dimension in type IIA (IIB) and the NS fields to the NS-branes. The classification of the $\mathrm{RR}$ fields has been an area of active research in the past several years. The charges can be classified, in the absence of the NS fields, by K-theory of spacetime [3], namely by $K^{0}(X)$ for type $I I B$ [4] and by $K^{1}(X)$ for type $I I A$ [5]. The RR fields are also classified by K-theory [6, 7], with the roles of $K^{0}$ and $K^{1}$ interchanged. In the presence of the NS B-field, or its field strength $H_{3}$, the relevant K-theory is twisted K-theory, in the sense of [8,9], as was shown in $[10,11]$ by analysis of worldsheet anomalies for the case the NS field $\left[H_{3}\right] \in H^{3}(X, \mathbb{Z})$ is a torsion class, and in [12] for the nontorsion case. Other versions of K-theory show up as well. For example, KO and KSp theories are relevant for Type I [4], equivariant K-theory for orbifolds, Atiyah's Real K-theory for orientifolds $[13,14]{ }^{1}$

Eleven-dimensional M-theory [16, 17, 18] has four BPS objects: the membrane M2, the fivebrane M5, the Kaluza-Klein monopole MKK and the gravitational wave MW. Compactification on a circle leads to type IIA string theory. The objects in the theory, i.e. the M-branes reduce to the IIA D-branes.

D-branes and M-branes can have anomalies associated to them. This prevents the existence of nonzero or well defined partition functions. For the M5 brane $[19,20]$, the partition function ${ }^{2}$ is nonzero if the M5 can be decoupled from the bulk, including the M2 brane. In type IIA, the D-brane anomaly cancellation [10] is the vanishing of the third integral Stiefel-Whitney class $W_{3}$. In the presence of a NS field, it becomes $\left[H_{3}\right]-W_{3}=0$. This can be interpreted in the context of (twisted) K-theory Atiyah-Hirzebruch spectral

\footnotetext{
${ }^{1} \mathrm{~A}$ review of early developments can be found in [15].

${ }^{2}$ Hopkins and Singer [21] have analyzed the M5 partition function, and constructed the line bundle on the intermediate Jacobian even when the fourth integral cohomology has torsion. Diaconescu, Freed and Moore [22] have also recently analyzed the M5 anomaly in the context of their model for the M-theory 3 -form $C_{3}$.
} 
sequence (AHSS) as the vanishing of the third differential $d_{3}$. This contains a topological part $W_{3}$ and a "twisting" part, the NS 3-form $H_{3}$.

On the other hand, there is another anomaly found by Diaconescu, Moore and Witten [1] in the context of relating the K-theory of ten-dimensional spacetime $X$ of type IIA to partition function of M-theory on an elevendimensional manifold $Y$ in the topological sector captured by an $E_{8}$ gauge theory. This anomaly is an obstruction to the partition function being well-defined. The condition is the vanishing of the seventh integral StiefelWhitney class $W_{7}$ of $X$, considered as the base of a circle bundle with total space $Y$. In [1] the NS fields were set to zero for the most part. In [23] flat NS potentials were added, including a (cohomologically trivial) NS field $H_{3}=d B_{2}$. However, the authors did not discuss the anomaly issue in that paper. Later, in [24], it was shown that the anomaly would still persist in the twisted K-theory setting.

A completely different motivation is to what extent K-theory can describe D-branes, and in particular the fields and the charges, and whether one should seek alternatives in some cases where K-theory breaks down and/or can not see the whole picture. For example, on Calabi-Yau spaces there currently seems to be an alternative: the derived category of coherent sheaves [25], and in the presence of a NS field, the derived category of twisted sheaves [26]. The advantage of using the derived category picture of $D$-branes as opposed to K-theory, is that the former picture contains considerably more data ${ }^{3}$ that specifies the D-brane (e.g. [27]). On non-geometric backgrounds, namely discrete-torsion D-branes there is a search for a "quantum K-theory" [28], and some other form of generalized cohomology is already being of relevance: $\mathbb{R} / \mathbb{Z}$ generalized cohomology theory ( $c f$. [29]). It is natural then to suspect that on general topological spaces an alternative of K-theory is needed, for example to describe phenomena at strong coupling and at the quantum level, perhaps more "M-theoretic" in nature.

In the present paper, we study the role of a new possible tool for investigating the $M$-theory partition function, namely elliptic cohomology. The first motivation which led us to consider elliptic cohomology was the question whether there is a twisting part that can be added to the topological part $W_{7}$ in analogy to $H_{3}$ in the brane-anomaly. The immediate candidate

\footnotetext{
${ }^{3}$ e.g. related to geometry and $D$-brane processes.
} 
would be the Hodge dual $H_{7}=*_{10} H_{3}$ of $H_{3}$. One might also naively ask whether $W_{7}$ analogously comes from a differential of degree $7, d_{7}$ in AHSS. But in K-theory AHSS of a 10-dimensional Spin manifld, $d_{7}$ is zero as can be seen from the following simple arguement. The AHSS is double-graded, $E_{p, q}$, where $p$ is the filtration degree (which is in the range 0 to 10 , the dimension of the manifold), and is 2-periodic in the $q$-direction. Thus, only even $q$-degrees occur and negative $q$-degrees occur as well. Now a differential $d_{r}$ raises the $p$-degree by $r$, and decreases the $q$-degree by $r-1$. Hence, $r$ must be odd. In any case, if the $p$-degree is to be raised by 7 , the only possible sources are 1 and 2 (the degrees 0 and 10 must be unaffected by differentials, since by K-theory valued Poincaré duality these K-theory classes survive). So $d_{7}$ in the cohomology K-theory AHSS can originate only in dimension 1 or 2 . But in dimension 1, every integral cohomology class is represented by a K-theory class. This is because $H^{1}(M, \mathbb{Z})$ classifies homotopy classes of maps $M \rightarrow S^{1}$, and the K-theory of $S^{1}$ is a free module on one (odd) generator, which we can pull back to $\mathrm{M}$. But a similar argument also works in dimension 2: $H^{2}(M, \mathbb{Z})$ is represented by a map $M \rightarrow \mathbb{C} P^{\infty}$ and the $\mathrm{K}$-theory cohomology AHSS collapses for $\mathbb{C} P^{\infty}$, in particular the generator of $H^{2}\left(\mathbb{C} P^{\infty}, \mathbb{Z}\right)$ is represented by a K-theory class, so its pullback represents the 2-dimensional integral cohomology class in $\mathrm{M}$. So $W_{7}$ can not possibly come from K-theory AHSS. However, the question will turn out not to be that naive and we will show that it indeed comes from a $d_{7}$, not in K-theory AHSS, but rather in Morava K-theory and elliptic cohomology! (Those are examples of generalized cohomology theories, which we explain in Appendix B.)

More concretely, we noticed (see Sections 3.2, 5.1 below) that the vanishing of the Diaconescu-Moore-Witten obstruction $W_{7}=0$ is precisely equivalent to orientability of the Spin-manifold $X$ with respect to (complexoriented) elliptic cohomology. This is a generalized cohomology theory which, in addition to the Bott-periodicity element $v_{1}$, contains another periodicity element $v_{2}$ of dimension 6 . Moreover, $v_{2}$ is invertible in elliptic cohomology, while $v_{1}$ is not.

This led us to ask questions: Is there an analogue of the $M$-theory partition function based on elliptic cohomology instead of $K$-theory? If so, what additional physical information does it contain? Is elliptic cohomology, rather than $K$-theory, the right tool for describing the path from $I I A$-theory 
to $M$-theory?

We do not have complete answers to all these question, but we do obtain clues for some of them. We do define, in Section 4 below, a lift of the partition function of [1] to elliptic cohomology. We focus on the compactified case $Y=X \times S^{1}$, in which case [1] show that the $M$-theory partition function is equivalent to that of IIA. We prefer, in fact, to work on the IIA-side of [1], as that is where K-theory "lives" 4. We show that the partition function construction in Section 7 of [1] can be lifted to elliptic cohomology. To be completely precise, this still requires that a Spin-manifold with $W_{7}=0$ is orientable with respect to real elliptic cohomology; we explain the difference in Appendix B. As it turns out, orientability with respect to real elliptic cohomology requires another condition, $w_{4}=0$. When this condition is violated, there is an additional anomaly. A discussion of the anomaly from the mathematical point of view is given in Section 5.2 below.

Now it appears that the elliptic cohomology-based partition function may indeed be more closely tied to $M$-theory than the $K$-theory partition function. For one thing, one can see directly (Section 4) that the elliptic partition function, when it exists, is not anomalous. (The distinction between existence and lack of anomaly may appear to be a fine one, but the point is in the $K$-theory case, there is a condition of vanishing of a certain quadratic function on torsion - this leads to $W_{7}=0$. In the elliptic cohomology setting, $W_{7}=0$ is a condition required for orientability, but when satisfied, no other condition on torsion appears.) Additionally, in elliptic cohomology, one has $E_{0}=\mathbb{Z}\left[v_{1}^{3} v_{2}^{-1}\right]$, so the partition function can be thought of as a family of partition functions indexed by a parameter $v_{1}^{3} v_{2}^{-1}$. So one can ask what is the physical meaning of these additional modes? The interpretation of $v_{2}$ as element in the 6-dimensional complex cobordism group suggests that these modes are related to interactions between an $M 2$-brane and $M 5$-brane. Out of the many possible configurations allowed by supergravity, we think the right ones are intersections between an $M 2$-brane and $M 5$-brane on a string, which, in the $S^{1}$-compactified case connects with the fundamental IIA-string. Moreover, this suggests that the theory can indeed be $H_{7}$-twisted, namely when the $M 5$-brane is not complex-oriented. Even in the untwisted case, however, we are seeing an anomaly of such states when $w_{4} \neq 0$. We also

\footnotetext{
${ }^{4}$ there one has the fundamental string mode expansion to work with, as we explain in Section 6 .
} 
think that the non-invertibility of the Bott element in elliptic cohomology is related to the disappearance of some of the $\mathrm{RR}$ charges when we pass from IIA to $M$-theory: these charges can be constructed one from another in any theory in which the Bott element is invertible. These ideas are given in Section 6 below.

The present paper relies very heavily on the methods of [1]. To make the paper self-contained, we review these methods in Sections 2, 3.1. We also require quite a lot of information from homotopy theory. Orientations are reviewed in Appendix A and additional comments salient to the present investigation are given in Section 3.3. Generalized cohomology theories, complex orientations, and formal group laws are reviewed in Appendix B. Section 5.1 contains the conclusion of the argument that a Spin-manifold with $W_{7}=0$ is orientable with respect to elliptic cohomology, and Section 5.2 describes the even much more subtle story surrounding $w_{4}$.

\section{The Diaconescu-Moore-Witten anomaly}

To make this paper as self-contained as possible, in this section we outline (only the relevant) results that we need from Diaconescu-Moore-Witten [1]. M-theory is defined on a circle bundle $Y$ with Type $I I A$ on the base $X$. In M-theory there is an $E_{8}$ bundle so associated to it is the $p_{1} / 2$ class $\lambda$. In Type II $A$ there are the RR fields, which are reductions of that class, and are classified by (twisted) K-theory. The nontorsion parts of the partition functions are compared and the anomalies are identified.

\subsection{The M-theory side}

First let us look at the M-theory side (compactified to $X$ ). Stong has shown that $\tilde{\Omega}_{10}^{\text {spin }}(K(\mathbb{Z}, 4))=\mathbb{Z}_{2} \times \mathbb{Z}_{2}$. This implies that there are two independent $\mathbb{Z}_{2}$-valued invariants of the pair $(X, a), a \in H^{4}(X, \mathbb{Z})$ :

1. $v(a)=\int_{X} a \cup w_{6}=\int_{X} a \cup S q^{2} \lambda$, where the second equality is due to $\mathrm{X}$ being spin. This is a linear function

$$
v(a+b)=v(a)+v(b)
$$


2. In $8 k+2$ dimensions, index $D_{V_{\mathbb{R}}}$ is a topological invariant mod 2 , name $f(a)$.

$$
\begin{aligned}
f(a+b) & =f(a)+f(b)+\int_{X} a \cup S q^{2} b \\
& =f(a)+f(b)+\int_{X} S q^{2} a \cup b
\end{aligned}
$$

where $f(a)$ is not a linear function.

The form $Q(a, b)=f(a+b)-f(a)-f(b)$ is a homeomorphism from $\Omega_{10}(K(\mathbb{Z}, 4) \times K(\mathbb{Z}, 4))$ to $\mathbb{Z}_{2} . \quad Q$ vanishes if either $a$ or $b$ is zero. This implies that $Q$ is a homeomorphism to $\mathbb{Z}_{2}$ of the relative bordism group

$$
\Omega_{10}^{\text {spin }}(K(\mathbb{Z}, 4) \times K(\mathbb{Z}, 4), K(\mathbb{Z}, 4) \times\{*\} \times\{*\} \times K(\mathbb{Z}, 4))
$$

which is calculated in [1] to be $\mathbb{Z}_{2}$. One of them is $Q(a, b)=\int_{X} a \cup S q^{2} b$, which is nonzero, e.g. on $X=S^{2} \times S^{2} \times \mathbb{C} P^{3}$.

Now what if $a$ is a torsion class. Then $Q(a, b)$ is a torsion pairing

$$
T: H_{\text {tors }}^{k}(X, \mathbb{Z}) \times H_{\text {tors }}^{n-k+1}(X, \mathbb{Z}) \rightarrow U(1) .
$$

This is because $\beta\left(S q^{2} b\right)=S q^{1} S q^{2} b=S q^{3} b$ by Adem relations. Now $S q^{3} b$ is a torsion class, and so $Q(a, b)=T\left(a, S q^{3} b\right)$. The partition function vanishes unless $Q=T=0$. This implies the condition $S q^{3} \lambda=0$. Therefore [1], M-theory on a spin manifold of the form $X \times S^{1}$ is inconsistent if $W_{7} \neq 0$.

\subsection{The IIA side}

The relevant calculations here are K-theory calculations [1] (and similarly [24] for the twisted case). Recall that we have the mod 2 index $I(v)$ of the Dirac operator with values in a real vector bundle $V$. For any $v \in K O(X)$, one can define the mod 2 index $I(v)$ of the Dirac operator with values in $v$. For any $x \in K(X)$ one has $x \otimes \bar{x} \in K O(X)$, so one can define $j(x)=I(x \otimes \bar{x})$.

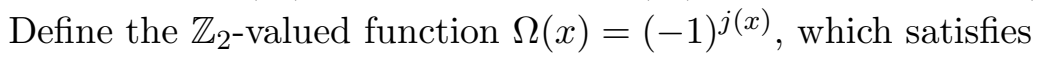

$$
\Omega(x+y)=\Omega(x) \Omega(y)(-1)^{\omega(x, y)}
$$

where $\omega(x, y)=I(x \otimes \bar{y})$ is an integer-valued unimodular antisymmetric bilinear form on the lattice $\Gamma=K(X) / K(X)_{\text {tors }}$. Now if $\Omega(x) \equiv 1$ for 
torsion elements of $K(X)$ then it can be regarded as a function on $\Gamma$ and so can be used to define the line bundle and hence its section, the RR partition function. If $\Omega(x) \not \equiv 1$ on $K(X)_{\text {tors }}$ then the Partition function of the theory vanishes upon summing over torsion. The story is similar in the twisted K-theory case [24] for $\Gamma_{H}=K(X ; H) / K(X ; H)_{\text {tors }}$.

Then [1] showed that the M-theory anomaly and the IIA anomaly are related in a one-to-one fashion:

$$
W_{7}(X) \neq 0 \text { if and only if } \Omega(x) \not \equiv 1 \text { on } K(X)_{\text {tors }} \text {. }
$$

The twisted case [24] is expected to result in an analogous statement.

\section{The Stiefel-Whitney classes}

\subsection{The $W_{3}$ story}

Freed and Witten [10] have shown that an anomaly in D-branes is given by:

$$
W_{3}+\left[H_{3}\right]=0
$$

$W_{3}$ is the integral class, obtained from the second mod 2 Stiefel-Whitney class $w_{2}$ via the Bockstein homomorphism. This is well-explained in [1] (or see the analogous construction for $W_{7}$ below). The physical interpretation of this is the following. A D-brane cannot wrap a submanifold of $X$ unless the Poincaré dual can be lifted to K-theory. The anomaly comes from the fact that when this condition is not satisfied then we can have other branes ending on the one we are considering and so we cannot view it in isolation, so the partition function is not well-defined (or -behaved).

There is more than one possible mathematical interpretation. At the level of AHSS, as we have seen in the introduction, it is the third differential $d_{3}=W_{3}+\left[H_{3}\right]$. At the level of the full twisted K-theory, one should solve the extension problem and this then could be an obstruction to a K-theory lift. There is another mathematical interpretation that will be relevant for our discussion of $W_{7}$. For a compact oriented manifold $\mathrm{X}$, this is an obstruction to being $\operatorname{Spin}^{c}$. But there is another way of looking at it, in terms 
of the K-theory AHSS differential. A Spin ${ }^{c}$-manifold would be K-theory orientable, so would have a K-theory homology fundamental class. Now one can see directly that if $W_{3} \neq 0, \mathrm{X}$ has no K-theory fundamental class. Stiefel-Whitney classes are conjugates of Steenrod operations by the Thom isomorphism. But one can also look at this in terms of Poincaré duality: Let $\alpha \in H^{7}(X, \mathbb{Z})$ (assuming $\mathrm{X}$ is 10 -dimensional) be a class such that $W_{3} . \alpha \neq 0$. Then

$$
S q^{3} \alpha \neq 0
$$

But now recall that the Milnor primitives ${ }^{5} Q_{i}$ are elements in the Steenrod algebra of dimension $2^{i+1}-1$, and that

$$
S q^{3}=Q_{1}+\text { decomposables. }
$$

Moreover, $Q_{1}$ is the primary differential $d_{3}$ in the K-theory AHSS. So we see that

$$
d_{3}(\alpha)=u
$$

in the $K^{*}$-AHSS, where $u \in H^{10}(X, \mathbb{Z})$ is the dual of the fundamental class. Dualizing, we conclude that the fundamental class of $\mathrm{X}$ does not lift to $\mathrm{K}$ theory homology, so X is not K-theory orientable.

\subsection{The $W_{7}$ story}

This section contains some observations regarding the obstruction $W_{7}(X)$, constructed in [1] for a compact Spin-10-manifold $X$, to the existence of a consistent theory of IIA RR $D$-branes in $X$, as well as to having a non-zero partition function of $M$-theory on $X \times S^{1}$.

We begin by recalling a subtlety regarding the definition of $W_{7}$ : this is not the 7-th Stiefel-Whitney class $w_{7} \in H^{7}(X, \mathbb{Z} / 2)$, which always vanishes for a Spin manifold. Rather, $W_{7} \in H^{7}(X, \mathbb{Z})$ is the canonical integral lift of $w_{7}$, namely

$$
\beta\left(w_{6}\right)
$$

where $\beta: H^{k}(X, \mathbb{Z} / 2) \rightarrow H^{k+1}(X, \mathbb{Z})$ is the Bockstein homomorphism. The fact that the $\bmod 2$ reduction $w_{7}$ of $W_{7}$ vanishes signifies the fact that $W_{7}$ is divisible by 2 in integral cohomology.

\footnotetext{
${ }^{5}$ see Appendix B for the definition and properties.
} 
This is strikingly analogous to another situation: namely, for a Spinmanifold $X$, the first Pontrjagin class $p_{1}$ is divisible by 2 , and $\lambda=p_{1} / 2$ is an obstruction to what $[30,31]$ call "string structure". One may ask if there is any connection between these two obstructions. One connection we can see right away. A string structure is the same thing as lifting the structure group of the tangent bundle of $X$ to the 3-connected cover String(10) of Spin(10). This is, however, by Bott periodicity the same thing as the 6-connected cover, as there are no homotopy groups in between. In other words, the classifying map $X \rightarrow B$ Spin(10) lifts to BString(10), which is 7-connected, and therefore has no cohomology in dimension 7 . Therefore, $W_{7}(X)=0$. Even more directly, one has $w_{6}=S q^{2} \lambda$, so $\lambda=0$ implies $w_{6}=0$ which implies $W_{7}=0$.

One may also ask if conversely, $W_{7}(X)=0$ implies $p_{1}(X) / 2=0$. However, a moment's reflection shows that this is false. For example, for $X=$ $S^{2} \times S^{2} \times \mathbb{C} P^{3}, p_{1} / 2$ is non-zero (and non-torsion), while there is no odd cohomology, so $W_{7}=0$.

We therefore further ask what is the geometric meaning of $W_{7} \neq 0$. To this end, recall the definition of Stiefel-Whitney classes: If we denote by $D: H^{k}(X) \rightarrow H_{10-k}(X)$ Poincaré duality, we have

$$
D\left(w_{k}\right)=S q^{k}(\mu)
$$

where $\mu \in H_{10}(X, \mathbb{Z} / 2)$ is the fundamental class. Here recall the action of Steenrod operations on homology $S q^{k}: H_{m}(X, \mathbb{Z} / 2) \rightarrow H_{m-k}(X, \mathbb{Z} / 2)$. We therefore have

$$
D\left(W_{7}\right)=\beta_{*} S q^{6}(\mu)
$$

where $\beta_{*}: H_{m}(X, \mathbb{Z} / 2) \rightarrow H_{m-1}(X, \mathbb{Z})$ is the Bockstein.

Now there is a distinguished integral cohomological operation

$$
\tilde{Q}_{2}: H^{m}(X, \mathbb{Z}) \rightarrow H^{m+7}(X, \mathbb{Z})
$$

(dually also a homological operation lowering dimension by 7) which is the integral lift of the Milnor primitive $Q_{2}$ (see [32], [33]). Moreover, the operation $\tilde{Q}_{2}$ is closely tied to a generalized cohomology theory $\tilde{K}(2)$ known as $p=2$ integral second Morava $K$-theory which is a reduction of elliptic cohomology (see [34] section 4.2, or Appendix B for background). One has

$$
\tilde{K}(2)^{*}(*)=\mathbb{Z}\left[v_{2}, v_{2}^{-1}\right]
$$


where $v_{2}$ is in dimension 6 . The way this theory is tied to $\tilde{Q}_{2}$ is as follows: as for every generalized cohomology theory, there is a corresponding generalized homology theory, and Atiyah-Hirzebruch spectral sequences both in homology and cohomology. Working, for example, in homology, the AHSS for $\tilde{K}(2)$ is

$$
E_{p q}^{2}=H_{p}\left(X, \tilde{K}(2)_{q}(*)\right) \Rightarrow \tilde{K}(2)_{p+q}(X) .
$$

The dimensions imply that possible differentials of this AHSS are $d^{6 k+1}$. The connection with $\tilde{Q}_{2}$ is that

$$
d^{7}=\tilde{Q}_{2}
$$

Now, moreover, $\tilde{Q}_{2}$ coincides with $\beta S q^{6}$ modulo elements of lower CartanSerre filtration (see [33]). Further, however, note that there are only three linearly independent Steenrod operations in dimension 6, namely ${ }^{6} S q^{6}$, $S q^{5} S q^{1}$ and $S q^{4} S q^{2}$. Further, on the fundamental class $\mu \in H_{10}(X, \mathbb{Z})$, the last two must vanish by the assumption that $X$ is a Spin manifold. We conclude that

$$
\tilde{Q}_{2}(\mu)=\beta S q^{6}(\mu)=D\left(W_{7}\right) .
$$

Therefore, we see that $W_{7} \neq 0$ if and only if the primary differential in the homology $\tilde{K}(2)$-AHSS for $X$ is non-zero on $\mu$, which in turn happens if and only if $X$ is not $\tilde{K}(2)$-orientable (as any higher differentials are out of filtration degree range). We have therefore proved

$$
\begin{gathered}
\text { A 10-manifold } X \text { is orientable with respect to } \tilde{K}(2) \text { if and only if } \\
W_{7}(X)=0 .
\end{gathered}
$$

\subsection{Orientation}

We have seen the two anomalies in ten dimensions, namely, $W_{3}=0$ the one coming from D-brane worldvolume, and $W_{7}=0$ the one coming from the M-theory partition function. Since orientation seems to be somewhat of a unifying theme for both anomalies, we will give some arguments on the relevance and possible consequences of having an orientation. In Appendix A, we have outlined some main points regarding orientations in any (generalized) cohomology. First let us consider the simplest case which is just the

\footnotetext{
${ }^{6}$ By Adem relations, one has $S q^{2} S q^{4}=S q^{6}+S q^{5} S q^{1}$ and $S q^{1} S q^{5}=0$.
} 
"usual" orientation of the manifold. One instance (aside from torsion issues) where orientation plays a role is in supersymmetry. For example, elevendimensional supergravity on $A d S_{4} \times N^{7}$ where $N^{7}$ is an Einstein manifold with killing spinors, can have different amount of supersymmetry depending on the orientation of $N^{7}$, and in some cases one of the two orientations leads to no supersymmetry at all.

One would like to be able to relate the cohomology of the branes to that of spacetime and vice versa. The Thom-Dold ${ }^{7}$ isomorphism and the Gysin homomorphism provide that link, without which it would be hard to study phenomena like anomaly inflow. A consequence of this is also the existence of Poincaré duality. This is desirable in order to be able to go from the description of D-branes as homology cycles and the description of fields and charges in term of cohomology. The formulae for the charges depends on characteristic classes, which are most elegant in the case when all the vector bundles are $E$-orientable. One consequence of viewing the problem as that of orientability is that certain properties follow immediately. For example, applying the theorem that says that any manifold is $E$-orientable if and only if its stable normal bundle is $E$-orientable, to the case $E=K$, one sees that the normal bundle being $\operatorname{spin}^{c}$ is a direct consequence of the manifold being so, and vice versa.

One can then define integers out of the characteristic classes, by pairing them with the fundamental class as in Appendix A. Of course, this is needed, e.g., to get integral charges for the branes. Another consequence is that one can use different orientations to get those charges. It seems reasonable then to believe that given an $E$-orientation of a manifold then one can find some integral formula, and changing the orietation to some $E^{\prime}$ then gives the charges in some other form, so we expect that the formula for the charges can be derived using Morava K-theory orientation and/or elliptic cohomology orientation. One might further suspect that the higher generalized cohomology theories could perhaps as well give corrections to the charge formula derived using K-theory.

Since the Steenrod square $S q$ is a ring morphism, one can apply (A.4)

\footnotetext{
${ }^{7}$ Dold generalized the Thom isomorphism and the Chern classes to generalized cohomology.
} 
to this case,

$$
\langle x,[X]\rangle=\langle S q(x) W(\nu),[X]\rangle,
$$

which when $\operatorname{dim} x \neq n$, i.e. the case relevant for the M-theory 4-form, reduces to ${ }^{8}$

$$
\langle S q(x) W(\nu),[X]\rangle=0
$$

Now when $S q^{1}(x)=0=S q^{2}(x)$, the condition becomes $S q^{3}(x) W^{3}(\nu)=0$. This implies that either $x$ has a K-theory lift [1] or that $W^{3}(\nu)$ vanishes, (or both of course).

One can relate orientations with respect to one cohomology theory to orienations with respect to another. For example, from the ring morphism $K O \rightarrow K$, any $K O$-orientable object is also $K$-orientable. This can be translated to the familiar language: every spin manifold is also $\operatorname{spin}^{c}$.

\subsection{Realizability}

The class $W_{3}$ can be realized by vector bundles, namely by the universal oriented vector bundle. If $W_{3}(\xi)=0$ for some $H \mathbb{Z}$-oriented vector bundle $\xi$, then $\xi$ is $K$-orientable. One possible consequence of this is the question of interpreting anomalies as obstruction to having a section on (determinant) bundles. In [10] the $W_{3}$ anomaly was interpreted using the line bundle Pfaff $(D) \otimes \mathcal{L}_{B}$ associated to the Dirac operator $D$ and the $B$-field. This suggests that the Diaconescu-Moore-Witten anomaly in M-theory cannot be represented in such a formalism. This hints that in the context of the $W_{7}$ anomaly, instead of (virtual) vector bundles, we should perhaps deal directly with classes in a generalized cohomology theory.

\section{Elliptic cohomology}

It is natural to conjecture that in the result of Section $3.2, \tilde{K}(2)$ can be replaced by elliptic cohomology $E$. This is in fact true, as can be proved by more technical manipulation of the AHSS (see Section 5.1). The first question one has to settle is which definition of $E$ we should choose. However, our characteristic classes observations give some clues. In particular, note

\footnotetext{
${ }^{8}$ All relations among those characteristic classes follow from this [35].
} 
that $W_{7}=0$ for any compact complex 10-manifold, so we should use complex oriented elliptic cohomology. On the other hand, recall that the HopkinsMiller universal elliptic cohomology theory tmf [36] is $M O\langle 8\rangle$-orientable, which means that every manifold whose stable normal bundle has structure group String $=O\langle 8\rangle$ is tmf-orientable. Thus, we have an explanation of the above distinction between the $p_{1} / 2$ and $W_{7}$ obstructions: it signifies that our current level of observation sees a connection between $M$-theory and complex-oriented elliptic cohomology, but not (yet) tmf.

Now there are still various models for complex-oriented cohomology $E$ which are characterized by their coefficient rings. Under certain basic assumptions (essentially, the associated elliptic curve must allow both multiplicative and $p=2$-supersingular reduction), these theories contain equivalent homotopical information, but each has some advantages or disadvantages. Choosing a complex-oriented elliptic cohomology theory is akin to choosing coordinates. For a fuller discussion, we refer the reader to Appendix B. The problem is that there cannot be a univeral complex-oriented elliptic cohomology theory. This is explained quite well in [37]. Although there is in some sense a universal (generalized) elliptic curve, called the Weierstrass curve given by the equation

$$
y^{2} x+a_{1} x y z+a_{3} y^{3}=x^{3}+a_{2} x^{2} z+a_{4} x z^{2}+a_{6} z^{3}
$$

over the ring $\mathbb{Z}\left[a_{1}, a_{2}, a_{3}, a_{4}, a_{6}\right]$, and we may choose accordingly

$E_{*}=\mathbb{Z}\left[a_{1}, a_{2}, a_{3}, a_{4}, a_{6}\right]\left[u, u^{-1}\right]$, this theory still cannot be considered universal because of automorphisms. However, the parameters $a_{i}$ are (generalized) modular forms, and there is a "character map"

$$
E \rightarrow K[[q]]\left[q^{-1}\right]
$$

where $K$ is $K$-theory, $q$ is a parameter of dimension $0, K[[q]]$ is therefore a product of infinitely many copies of $K$, and the notation $\left[q^{-1}\right]$ signifies that $q$ is inverted. The map (4.1) is determined by what happens on coefficients, which can be found for example in [37], Section 2.6, see also Appendix B.

The Weierstrass equation (and the definition of $E$ ) can be simplified substantially if the prime 6 is inverted. Unfortunately, this is not suitable for us, as $p=2$ information is critical to to our investigation (as seen already in the $K$-theory calculation of the IIA partition function described in [1]). Another kind of simplification arises if we are willing to complete at the 
prime 2. For example, we may then choose $E_{*}=W_{2}[[a]]\left[u, u^{-1}\right]$ where $W_{2}$ is the ring of Witt vectors, i.e. the ring of integers of the extension of the field $\mathbb{Q}_{2}$ of 2 -adic numbers by an Eisenstein polynomial of degree $4, \operatorname{dim}(a)=0$, $\operatorname{dim}(u)=2$. The characteristic property of this theory is that its formal group law, calculating $c_{1}(\xi \otimes \eta)$ from $c_{1}(\xi), c_{1}(\eta)$ for line bundles $\xi, \eta$ is a Lubin-Tate law $F_{2}$ of height 2 (see [38]). One can construct a character map (4.1) for this theory with the only exception that $K$ must be replaced by $K$-theory with coefficients in $W_{2}$ (see again Appendix B for more details).

An even further simplification may be obtained if we notice that the cohomology theory $E$ of the last paragraph (with $E_{*}=W_{2}[[a]]\left[u, u^{-1}\right]$ ) is a completion of a finite sum of suspensions of copies of the cohomology theory $E(2)$ which has $E(2)_{*}=\mathbb{Z}\left[v_{1}, v_{2}, v_{2}^{-1}\right]$ where $v_{1}$ has dimension 2 and $v_{2}$ has dimension 6 . One may set, for example, $v_{2}=u^{3}, v_{1}=a u$. This cohomology theory lacks some of the manifest modular symmetries of the other elliptic cohomology theories, (in particular is only 6-periodic in dimension), but has the simplest coefficients and we will often find it most convenient for our purposes.

One more refinement is relevant. Throughout our discussion, we are dealing with Spin manifolds, and hence some real structure is expected to play a role. Therefore, we need to consider a real form $E O(2)$ of elliptic cohomology, which is obtained, roughly speaking, by taking the fixed point cohomology theory of $E$ under $\mathbb{Z} / 2$-action which comes from the FGL isomorphism $-i(x)$ where $i(x)$ is the inverse series of $F_{2}$. In fact, making such definition precise is difficult, as one must prove the $\mathbb{Z} / 2$-action is rigid, and not just up to homotopy. However, a rigorous definition of $E O(2)$ is given in [39], and its coefficients $E O(2)_{*}$ are calculated there also. To construct our elliptic partition function, from now on, we shall assume that $X$ is orientable under $E O(2)$. One could conjecture this is true if and only if $W_{7}=0$ and $X$ is Spin, but it turns out that the situation is not quite as simple as that. Instead, a Spin manifold is $E O(2)$-orientable if and only if it satisfies $w_{4}=0$ (we prove this in Section 5.2 below). In other words, when $w_{4} \neq 0$, we see yet another anomaly to the existence of an elliptic cohomology partition function.

Now recall from [1] and Section 2.2 above that the key point of the construction of the RR paritition function of IIA $D$-branes is the function $\Omega$ (or 
equivalently $j$ ) defined on $K^{0}(X)$. Homotopy-theoretically, the construction of $j$ means that for $x \in K^{0}(X)$, the virtual bundle $x \otimes \bar{x}$ has a real structure, and hence represents an element of $K O^{0}(X)$. The mod 2 index is simply the Kronecker product with the $K O$-orientation $K O$-homology class $\mu \in K O_{10}(X)$ :

$$
K O^{0}(X) \otimes_{K O_{*}} K O_{10}(X) \rightarrow K O_{10}(*)=\mathbb{Z} / 2 .
$$

The construction of the partition function fails when this index is non-zero on torsion elements $x \in K^{0}(X)$.

Let us now mimic the construction of the theta-function described in [1], Section 7 in elliptic cohomology. To this end, as suggested in Section 3.4 above, we shall deal directly with generalized cohomology classes, in this case with $E^{0}(X)$-classes. The manifold $X$ is $E$-orientable, so it has an orientation class $[X]_{E} \in E_{10} X$. For $x, y \in E^{0}(X)$, we put

$$
\omega(x, y)=\left\langle x \bar{y},[X]_{E}\right\rangle \in E_{10}=E_{0}
$$

Now we need an elliptic refinement of the function $j$. Assuming that $X$ is $E O(2)$-orientable, we have an $E O(2)$-orientation class $[X]_{E O(2)} \in E O(2)_{10}(X)$. Now for $x \in E^{0}(X)$, the class $x \bar{x}$ lifts canonically to $E O(2)^{0}(X)$, so we may put

$$
j(x)=\left\langle x \bar{x},[X]_{E O(2)}\right\rangle \in E O(2)_{10} .
$$

To see what the right hand side is, we must compute $E O(2)_{10}$. This was done in [39]. It is helpful for the purpose of the computation to reduce $E$ to a theory $E(2)$ with coefficients $\mathbb{Z}_{2}\left[v_{1}, v_{2}, v_{2}^{-1}\right]$ (it is a direct summand of $E$, so this will allow us to omit repeating terms). Now one more relevant piece of information is the twist. The correct way of viewing the real version of the theory $E(2)$ is as a $\mathbb{Z} / 2$-equivariant generalized cohomology theory, which we denote by $E \mathbb{R}(2)$. Cohomology classes of such theory are indexed by $k+\ell \alpha$ where $\alpha$ denotes the sign representation of $\mathbb{Z} / 2$ (see [39]). Then the orientation class of $X$, which we assume, is in $E \mathbb{R}(2)_{10}(X)$, so the Kronecker product lies in $E \mathbb{R}(2)_{10}(*)$. In the notation of [39], this group is a $\mathbb{Z} / 2$-vector space with basis

$$
v_{1}^{3 n} v_{2}^{2-n} \sigma^{-4} a^{2}, n \geq 1
$$

Here $v_{1}$ has dimension $1+\alpha, v_{2}$ has dimension $3(1+\alpha)$, $\sigma$ has dimension $\alpha-1$ and $a$ has dimension $-\alpha$, so the generators (4.2) have dimension 10. See Appendix $\mathrm{B}$ for more on the theory $E \mathbb{R}(2)$. In any case, we have produced an element $j(x) \in \mathbb{Z} / 2\left[v_{1}^{3} v_{2}^{-1}\right]=E O(2)_{10}$. 
To be able to say that this element $j$ is an elliptic refinement of the element constructed in [1], i.e. to produce, upon a choice of the parameter $v_{2}$, a function $\Omega(x)$ with (2.6), we must make sense of the identity

$$
j(x)+j(y)-j(x+y) \equiv \omega(x, y) \quad \bmod 2 .
$$

Note that this is more delicate than in the $K$-theory case, as we do not (at least at present) have index-theoretical arguments at our disposal for $E(2)$, and the two sides of (4.3) apparently belong to different generalized cohomology groups. A purely homotopy-theoretical argument, however, is possible, and in fact its analogue is interesting in the case of $K$-theory, too. We see that the left hand side of (4.3) is

$$
\left\langle x \bar{y}+\bar{x} y,[X]_{E O(2)}\right\rangle,
$$

while the right hand side is

$$
\left\langle x \bar{y},[X]_{E(2)}\right\rangle .
$$

It will therefore suffice if we can make sense, for any $a \in E(2)^{0}(X)$, of the identity

$$
\left\langle a+\bar{a},[X]_{E O(2)}\right\rangle \equiv\left\langle a,[X]_{E(2)}\right\rangle \quad \bmod a+2 .
$$

To interpret (4.4), we consider the transfer map

$$
\tau: E(2) \rightarrow E O(2)
$$

In the notation of [39], this can be interpreted for example as a map on fixed points of the $\mathbb{Z} / 2$-equivariant generalized cohomology theories

$$
E \mathbb{R}(2) \wedge\left(\mathbb{Z} / 2_{+}\right) \longrightarrow E \mathbb{R}(2) \wedge\left(E \mathbb{Z} / 2_{+}\right) \stackrel{N}{\longrightarrow} F\left(E \mathbb{Z} / 2_{+}, E \mathbb{R}(2)\right) .
$$

Here $E \mathbb{Z} / 2$ is a contractible space with free $\mathbb{Z} / 2$-action. The second arrow $N$ of (4.5) is the norm map from a Borel homology to Borel cohomology theory (see [39]). Now for $a \in E(2)^{0} X$, we have the following commutative diagram:

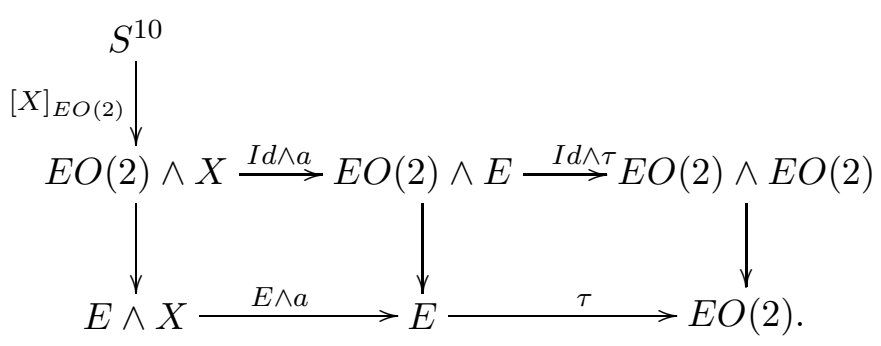


The unlabelled arrows are the forgetful map and multiplications. Now this diagram shows that (4.4) is valid if we map the right hand side to the left hand side using the transfer! But is the transfer non-trivial on the elements concerned? This is where (4.5) comes in. It is shown in [39] that the norm map can be calculated by dividing by $\sigma a$ the differential in the Tate cohomology spectral sequence for $\widehat{E R}$ which crosses the line between Borel homology and cohomology. The relevant differential is

$$
d: \sigma^{-2} \mapsto v_{1} a^{3},
$$

so we get for $n \geq 1$,

$$
v_{1}^{3 n} v_{2}^{2-n} \sigma^{-4} a^{2}=\tau\left(v_{1}^{3 n-1} v_{2}^{2-n} \sigma^{-5}\right) .
$$

On the right hand side, we are in $E(2)_{10}$, so the $\sigma$ 's can be dropped. We see these are precisely the generators of $E(2)_{10}$.

Thus, we have produced an analogue of the setup of [1], Section 7.1, but over the ring $\mathbb{Z}\left[v_{1}^{3} v_{2}^{-1}\right]$ instead of $\mathbb{Z}$. We would like to interpret this as a family of theta-functions parametrized by $v_{1}^{3} v_{2}^{-1}$. The question is, can this parametric theta-function be anomalous and how is it related to the theta-function of [1], since we are using a different generalized cohomology theory?

To answer the first question, it turns out that this theta-function cannot be anomalous, although it is possible that it could be twisted, see Section 6 below. To show that no anomaly is possible, note that anomaly of the construction [1], Section 7.1 can arise when the function $j$ is non-zero on torsion elements. We have seen that this cannot happen when $X$ is $E$ orientable (see also a more direct argument below). However, this is not quite enough, as a priori additional anomaly could be related to torsion in the element $v_{1}^{3} v_{2}^{-1}$. Fortunately, this does not happen: we will see in Section 5.1 below from AHSS arguments that

$$
E^{0}(X)=k^{0}(X)\left[v_{1}^{3} v_{2}^{-1}\right]=K^{0}(X)\left[v_{1}^{3} v_{2}^{-1}\right]
$$

so there is no torsion in the $v_{1}^{3} v_{2}^{-1}$ element.

To address the second question (comparison of theta-functions), we invoke the character map (4.1). By (4.6), we see that the theta-function we 
construct must be but a 1-parametric family of ordinary elliptic functions in the parameter $q$, by the comparison map from elliptic cohomology to $K$-theory. Discarding the additional parameter, therefore, we obtain the partition function of [1].

To give a direct argument for vanishing of the $K$-anomaly in the $E$ partition function in terms of bundles, consider again a complex bundle with real structure $x \otimes \bar{x}$. Now this cannot be identified with an element of $E O(2)^{*}(X)$. On the other hand, bundles with real structure have characteristic classes in $E O(2)^{*}(X)$. Which characteristic class should we choose? There is such characteristic class for every polynomial in Chern classes. The description of that class we choose depends in part on our complex orientation, i.e. on the formal group law on $E_{*}$ : let us therefore assume, without loss of generality, that the law, upon reduction to Morava $K(2)$-theory, has

$$
x+{ }_{F_{2}} x=2 x+u^{3} x^{4} .
$$

(This means that the elliptic cohomology has an automorphism which turns the Lubin-Tate law into the FGL, which after reduction to Morava $K(2)$ theory, is (4.7).) Now Chern classes are, in any complex-oriented cohomology, determined by their values on direct sums of line bundles. The class we are interested in is the determinant class $D$ given by

$$
D\left(L_{1} \oplus \ldots \oplus L_{n}\right)=c_{1}\left(L_{1}\right)+{ }_{F_{2}} \ldots{ }_{F_{2}} c_{1}\left(L_{n}\right) .
$$

As remarked above, for a complex bundle with real structure we automatically get a corresponding $E O(2)^{*}(X)$-valued characteristic class. Now since we are assuming $X$ is $E O(2)$-oriented, let $[X]_{E O(2)} \in E O(2){ }_{10} X$ again be the orientation class. We may therefore go ahead and define $j_{E}(x) \in E O(2)_{*}$ by the formula

$$
j_{E}(x)=\left\langle D(x \otimes \bar{x}),[X]_{E O(2)}\right\rangle .
$$

The map (4.1) shows that the reduction of $j_{E}$ to $K$-theory is the invariant $j$ considered above.

It is appropriate here to make note that we have introduced an $\alpha$ twist. The determinant characteristic class, which we selected, now lands in $E \mathbb{R}(2)^{1+\alpha}(X)$ where $\alpha$ denotes the sign representation of $\mathbb{Z} / 2$ (see [39]). Then the orientation class of $X$, which we assume, is in $E \mathbb{R}(2)_{10}(X)$, so the Kronecker product lies in $E \mathbb{R}(2)_{9-\alpha}(*)$. In the notation of [39], this group 
is a $\mathbb{Z} / 2$-vector space with basis

$$
v_{1}^{3 n-1} v_{2}^{2-n} \sigma^{-4} a^{2}
$$

Here $v_{1}$ has dimension $1+\alpha, v_{2}$ has dimension $3(1+\alpha)$, $\sigma$ has dimension $\alpha-1$ and $a$ has dimension $-\alpha$, so the generators (4.9) have dimension $9-\alpha$.

Now it can be seen directly that the invariant $j_{E}$ vanishes on torsion elements of $K^{0}(X)$. To see this, consider the formula (4.7) above. Reducing modulo 2, the first summand vanishes. Reducing further to Morava $K$ theory [46], taking $2^{n}$-th powers is an automorphism of the theory, and $u$ is invertible. Consequently, by completeness, the same conclusion must hold for $E O(2): j_{E}(2 x)$ for a bundle $x$ is just an image of $j_{E}(x)$ under an automorphism of the theory, hence cannot vanish if $j_{E}(x)$ does not vanish. However, if $2^{k} x=0$ (the only interesting torsion is at the prime 2), then $j_{E}\left(2^{k} x\right)$ must vanish, hence so does $j_{E}(x)$ !

It is curious that the lifting of bundles to elliptic cohomology which showed the vanishing of $j_{E}$ on twisting is non-linear, and instead has a degree 4 correction, coming from (4.7). We saw above that the right approach is to work in elliptic cohomology directly, but it would be nice to have a direct physical interpretation of this non-linear behavior on bundles.

From the point of view of $M$-theory, it is interesting to note that $p_{1} / 2$ is the obstrution for the $L K(\mathbb{Z}, 3$ )- (hence $L E(8)-$ ) bundle on $X$ induced from the tangent bundle on $Y=X \times S^{1}$ to lift to a $\tilde{L} E(8)$-bundle where $\tilde{L} E(8)$ denotes the universal central extension (= the "affine group"). If such bundle existed ${ }^{9}$, then again $X$ is orientable with respect to $t m f$. From the above discussion, we see once again that orientability with respect to $E O(2)$ is the more salient notion at the current level of observation.

More concretely, recall again that the phase factor $f(a)$ of $a \in H^{4}(X, \mathbb{Z})$ can be calculated by taking mod 2 index of the adjoint $e_{8}$-bundle $\xi$ associated with $a$. This can again be written by considering $\xi \in K O^{0}(X)$; the $\bmod 2$ index is then the Kronecker product with the fundamental class $\mu \in K O_{10}(X)$. Therefore, when $X$ is $E O(2)$-orientable, we can again refine this invariant

\footnotetext{
${ }^{9}$ This was considered in [24].
} 
to elliptic cohomology, i.e. put

$$
f_{E}(a)=\left\langle x,[X]_{E O(2)}\right\rangle \in E O(2)_{*}
$$

where $x$ is a lift of $\xi$ to elliptic cohomology. It can therefore be expected that the entire elliptic theta-function can be seen also from the $M$-theoretical point of view, although a careful comparison between both sides is needed (see Section 6 for more on this).

This now points to a connection with the speculations [45] that elliptic cohomology index can be calculated as some version of index of the Dirac operator on the associated loop bundle. Therefore, instead of an $E_{8}$-bundle, we are compelled to consider the associated $L E_{8}$-bundle. That bundle resides on $L X$, but [45] gives evidence that the index information may be concentrated around constant loops. In that case, an $L E_{8}$-bundle on $X$, again, corresponds to an $E_{8}$-bundle on $X \times S^{1}$. The line of thought of [45] is that the $t m f$-index of $\xi$ (for example the Witten genus) would be calculable in this geometric way if $p_{1} / 2(X)=0$. The evidence from the $M$-theory partition function which we have gathered points to the conclusion that the corresponding $E O(2)$-index should have such geometric interpretation if and only if $X$ is Spin and $w_{4}(X)=0$. It is also worth noting that a direct connection of bundles on loop space with elliptic cohomology, via 2-vector bundles and $K$-theory of $k u$, was proposed in [47].

\section{$5 \quad$ Orientability with respect to elliptic cohomology}

\subsection{The E story}

In this section, we prove that a Spin-manifold $X$ with $W_{7}=0$ is orientable with respect to complex-oriented elliptic cohomology $E$. As explained above, instead of $E$, we may work with $E(2)$ which has coefficients $E(2)_{*}=\mathbb{Z}_{2}\left[v_{1}, v_{2}, v_{2}^{-1}\right]$ where $\operatorname{dim}\left(v_{1}\right)=2, \operatorname{dim}\left(v_{2}\right)=6$.

To start the proof, first note that we already know that $X$ is orientable with respect to $K$-theory. We next claim that $X$ is also orientable with respect to connective $k$-theory. To see this, consider the $k$-theory homology AHSS

$$
E_{p q}^{2}=H_{p}\left(X, k_{q}(*)\right) \Rightarrow k_{p+q} X
$$


We have $k_{*}(*)=\mathbb{Z}\left[v_{1}\right]$. The proof uses the observation that, in fact for every space $X$, in the spectral sequence (5.1), we have

$$
v_{1}: E_{*, q}^{r} \rightarrow E_{*, q+2}^{r} \text { is onto for all } q \text { and iso for } q \geq r-2 \text {. }
$$

This is proved by induction on $r$. It is true for $r=2$. So assume (5.2) is true for a particular $r$, we try to prove it with $r$ replaced by $r+1$. Thus, consider

$$
d^{r}: E_{p, q}^{r} \rightarrow E_{p-r, q+r-1}^{r} .
$$

First, if $d^{r}\left(v_{1} x\right)=v_{1} d^{r}(x)=0$, then $d^{r}(x)=0$ by the induction hypothesis, so $\operatorname{Ker}\left(d^{r}\right)$ is generated in $q$-degree 0 , and hence so is $H\left(d^{r}\right)=E^{r+1}$, proving the "onto" part of the statement with $r$ replaced by $r+1$. To prove the "iso" part, consider $x \in E_{*, \geq r}^{r}$ with $v_{1} x=d^{r} y$. Then $y=v_{1} z$ for some $z$ by the induction hypothesis. So $v_{1}\left(x-d^{r} z\right)=0$, but then $x-d^{r} z=0$ by the induction hypothesis, concluding the proof of the "iso" part.

But now from (5.2), it follows that whenever $d^{r}(x) \neq 0$ in the spectral sequence (5.1), it is also non-zero in the spectral sequence obtained from (5.1) by inverting $v_{1}$, which is the $K$-theory homology AHSS. In particular, since $X$ is $K$-orientable, its homology fundamental class supports no differential in the $K$-theory homology AHSS, hence by what we just said supports no differential in the $k$-theory AHSS, hence $X$ is $k$-orientable.

Now assume as we did that $X$ has, in addition, $W_{7}=0$. As observed above, then $X$ is $\tilde{K}(2)$-orientable where $\tilde{K}(2)$ is integral Morava $K$-theory with $\tilde{K}(2)_{*}=\mathbb{Z}\left[v_{2}, v_{2}\right]^{-1}$. This was simply because by sparsity of dimensions, the only possible differential on the fundamental class was $d_{7}$ which was identified with the class $W_{7}$. Hence, the exact same argument also proves that $X$ is orientable with respect to the connective integral Morava $K$-theory $\tilde{k}(2)$, which has $\tilde{k}(2)_{*}=\mathbb{Z}\left[v_{2}\right]$. In fact, we claim that we can prove a stronger statement, namely that

$$
\text { The } \tilde{k}(2) \text {-homology AHSS for } X \text { collapses to } E^{2} \text {. }
$$

To prove this, note that since we already know $X$ is $\tilde{k}(2)$-orientable, the $\tilde{k}(2)$-homology and cohomology AHSS's are isomorphic (by capping with the fundamental class, which is a permanent cycle). So we can work in the cohomology AHSS instead. Now by sparsity of dimensions, the first possible differential is $d_{7}$. Since $d_{7}$ cannot disturb filtration degrees 0 and 10 (by orientability), the only possible differentials are $d_{7}: E_{2}^{1, *} \rightarrow E_{2}^{8, *}$ or 
$d_{7}: E_{2}^{2, *} \rightarrow E_{2}^{9, *}$. But now first and second integral ordinary cohomology classes are represented by maps into $S^{1}$ and $\mathbb{C} P^{\infty}$ respectively, for which the $\tilde{k}(2)$-cohomology AHSS collapse. Therefore, by naturality of spectral sequances, the $d^{7}$ 's considered must also be 0, concluding the proof of (5.4).

Now we shall consider the second Johnson-Wilson generalized cohomology theory $B P\langle 2\rangle$ (see [34], Section 4.2) with coefficients $B P\langle 2\rangle_{*}=\mathbb{Z}\left[v_{1}, v_{2}\right]$. We have a cofibration sequence of generalized cohomology theories (more precisely spectra)

$$
v_{1} \cdot B P\langle 2\rangle \rightarrow B P\langle 2\rangle \rightarrow \tilde{k}(2) .
$$

This leads to an exact couple, and a corresponding spectral sequence

$$
E_{p, q}^{2}=\tilde{k}(2)_{p} X \otimes k_{q} \Rightarrow B P\langle 2\rangle_{p+q} X
$$

(Recall that $k_{*}=\mathbb{Z}\left[v_{1}\right]$ where $v_{1}$ is in dimension 2.) Also, we have just proved that

$$
\tilde{k}(2)_{*} X=H_{*}(X, \mathbb{Z}) \otimes \tilde{k}(2)_{*} .
$$

Now we would like to claim that

The fundamental class $\mu \in \tilde{k}(2)_{10} X$ is a permanent cycle in (5.6).

To this end, first note that there is a comparison map from the cofibration (5.5) to the cofibration sequence

$$
v_{1} k \rightarrow k \rightarrow H \mathbb{Z}
$$

and hence a comparison map from (5.6) to (5.1). This means that any target of a differential on $\mu$ in (5.6) must be of the form $v_{2} a$ (as otherwise the comparison map would give a differential in (5.1), which has no differential originating in $\mu$ ). But now dimensional considerations show that the only possibility is

$$
d_{3}(\mu)=v_{1} v_{2} a
$$

for some $a \in H_{1}(X, \mathbb{Z})$. Note that translating this back to cofibration sequences, this means that the connecting map

$$
\beta: \tilde{k}(2)_{10} X \rightarrow B P\langle 2\rangle_{7} X
$$

satisfies

$$
\beta(\mu)=v_{2} a
$$


for some $a \in B P\langle 2\rangle_{1} X$ which, moreover, has non-trivial reduction in $H_{1}(X, \mathbb{Z})$. Note that this implies that

$$
v_{1} v_{2}(a)=0
$$

(as $\beta$ is the connecting map of $v_{1}$ ). Now we distinguish two cases. First, suppose $a$ is non-torsion. Then there exists a cohomology class $u \in H^{1}(X, \mathbb{Z})$ such that $\langle a, u\rangle \neq 0$. But then (5.13) implies that $u$ cannot lift to $B P\langle 2\rangle^{1} X$, which contradicts the fact that the $B P\langle 2\rangle$-cohomology AHSS collapses on $S^{1}$ (which represents $H^{1}(X, \mathbb{Z})$ ). The second case is that $a$ is torsion, but then there is a class $u \in H^{2}(X, \mathbb{Z})$ which is the Bockstein of a class $v \in$ $H^{1}(X, \mathbb{Z} / m)$ such that $\langle a \bmod m, v\rangle \neq 0$. Similarly as above, however, we see from (5.13) that $u$ cannot lift to $B P\langle 2\rangle^{2} X$, which is again a contradiction, as $H^{2}(X, \mathbb{Z})$ is represented by maps to $\mathbb{C} P^{\infty}$, and the $B P\langle 2\rangle$ cohomology AHSS collapses for $\mathbb{C} P^{\infty}$. This concludes the proof of (5.8).

Therefore, we have shown that $X$ is orientable with respect to $B P\langle 2\rangle$. But we have a comparison map of ring spectra

$$
B P\langle 2\rangle \rightarrow E(2)
$$

(which is inclusion on coefficients), and therefore $X$ is $E(2)$-orientable, as claimed.

\subsection{The $E O(2)$ story}

In this section, we will prove the following:

A Spin-manifold $X$ is orientable with respect to $E O(2)$ if and only if it satisfies $w_{4}=0$.

We first focus on the necessity of the condition. As remarked above, $E O(2)$ can be considered the spectrum of fixed points (or, in this case equivalently, homotopy fixed points) of the equivariant cohomology theory $E \mathbb{R}(2)$, which is a $\mathbb{Z} / 2$-equivariant version of $E(2)$. The $\mathbb{Z} / 2$-equivariant real elliptic cohomology spectrum was considered extensively in [39]. Now the primary obstruction to orientability with respect to $\mathbb{Z} / 2$-equivariant integral Morava $K(2)$-theory $\tilde{K} \mathbb{R}(2)$ is

$$
\mathbb{Q}_{2}[X]
$$


where $\mathbb{Q}_{2}$ is the second real Milnor primitive (more precisely, we should be considering again the integral version of the Milnor primitive, but for the purposes of necessity, if the integral element vanishes, so does its $\bmod 2$ reduction). The story of the $\mathbb{Z} / 2$-equivariant Steenrod algebra is told extensively in [39], Section 6. It turns out that the dual of this algebra can be described in two equivalent ways: either

$$
A_{\star}^{c}=\mathbb{Z} / 2\left[\zeta_{i}, a, \sigma, \sigma^{-1}\right]
$$

where $\operatorname{dim}\left(\zeta_{i}\right)=2^{i}-1, i=1,2, \ldots, \operatorname{dim}(a)=-\alpha, \operatorname{dim}(\sigma)=1-\alpha($ see Appendix B). The other description is as

$$
A_{\star}^{c}=\mathbb{Z} / 2\left[\xi_{i}, \tau_{i}, a, \sigma, \sigma^{-1}\right]
$$

where now $\operatorname{dim}\left(\xi_{i}\right)=(1+\alpha)\left(2^{i}-1\right), i \geq 1, \operatorname{dim}\left(\tau_{i}\right)=1+(1+\alpha)\left(2^{i}-1\right), i \geq 0$. In this second description (5.17), we then have a basis of the $\mathbb{Z} / 2$-equivariant Steenrod algebra $A_{c}^{\star}$ which is the $\mathbb{Z} / 2[[a]]\left[\sigma, \sigma^{-1}\right]$-basis dual to

$$
\prod \tau_{i}^{\epsilon_{i}^{i}} \prod \xi_{i}^{p_{i}^{*}}
$$

(where $\epsilon_{i}=0,1, r_{i}=0,1,2, \ldots$ and all but finitely many of these values are $0)$. This dual basis can be written as

$$
\prod \mathbb{Q}_{i}^{\epsilon_{i}} \prod \mathbb{P}^{\left(r_{1}, r_{2}, \ldots\right)}
$$

The elements $\mathbb{Q}_{i}$ (of dimension $1+(1+\alpha)\left(2^{i}-1\right)$ ) are the real Milnor primitives. On the other hand, using the description (5.16), also the usual $p=2$-Milnor basis

$$
S q^{R}
$$

serves as a $\mathbb{Z} / 2[[a]]\left[\sigma, \sigma^{-1}\right]$-basis of $A_{c}^{\star}$.

Now the key to understanding the obstruction (5.15) is converting the element $\mathbb{Q}_{2}$ to the basis (5.20). In particular, we claim that one of the summands of $\mathbb{Q}_{2}$ is

$$
S q^{(4,0, \ldots)} a^{3}
$$

To show that is equivalent to expanding $\zeta_{1}^{4}$ in the basis (5.18). Using the recursive formulas given in [39], Section 6 , we find that

$$
\begin{aligned}
& \zeta_{1}^{4} \equiv \tau_{2} a^{3}+\xi_{2} \sigma^{-1} a^{2}+\xi_{1}^{2} \sigma^{-2}+\xi_{2} \tau_{0} a^{3} \\
& +\xi_{1}^{2} \tau_{1} a^{3}+\xi_{1}^{3} \sigma^{-1} a^{2}+\xi_{1}^{3} \tau_{0} a^{3} \bmod a^{4},
\end{aligned}
$$


so we see that $\tau_{2} a^{3}$ is present, which proves that (5.21) is present in the expansion of $\mathbb{Q}_{2}$. This proves that $a^{3} S q^{(4,0, \ldots)}[X]$ is a summand of (5.15), but for a Spin-manifold,

$$
S q^{(4,0, \ldots)}[X]=S q^{4}[X]
$$

which is Poincaré dual to $w_{4}$. This concludes the proof of necessity of our condition.

Sufficiency follows from the advanced theory which Ando-Hopkins-Rezk [40] developed to treat orientability with respect to $t m f$. We give the argument here merely for completeness. One starts with the concepts of MayQuinn-Ray [44]: For an $E_{\infty}$ ring spectrum $E$ (a multiplicative generalized cohomology theory with a particularly nice multiplication), one can construct an infinite loop space of units of $E$ which [40] denote by $G L_{1}(E)$ ([44] use the notation $E^{\otimes}$ ). As a space, (i.e. forgetting infinite loop space structure), $G L_{1}(E)$ is simply the disjoint union of the connected components of the infinite loop space associated with $E$ which are invertible under multiplication in $\pi_{0}(E)$.

Now infinite loop spaces correspond essentially bijectively with connective spectra (infinite loop space theories whose coefficients are 0 in negative degrees). A convention of [40] assigns to an infinite loop space written in capital letters the connective spectrum with the same notation but in lower case letters; for example, the connective spectrum corresponding to $G L_{1}(E)$ is $g l_{1}(E)$.

Now Rezk [41], following Kuhn [42], defines a map

$$
\phi: g l_{1}\left(E_{2}\right) \rightarrow E_{2}
$$

(More generally, he defines for any $E_{\infty}$-ring spectrum $E$ a map $g l_{1}(E) \rightarrow$ $L_{K(n)}(E)$.) Now recall from the last paragraph that for $q>0$,

$$
\pi_{q}\left(g l_{1}(E)\right) \cong \pi_{q}(E)
$$

Therefore, one may ask in that notation what the map $\phi$ of (5.22) induces on homotopy groups. Rezk gives a complete formula in [41], which goes as follows (working at the prime 2): For an element $x \in\left(\widetilde{E_{2}}\right)^{0} S^{2 k}$ (reduced 
$E_{2}$-cohomology), one has

$$
\phi_{*}(x)=x-\frac{1}{2} \sum_{i=1}^{3} \psi_{\alpha_{i}}(x)+\psi_{2}(x)
$$

where $\psi_{\alpha_{i}}, \psi_{2}$ are Ando's power operations [43]. Before explaining them, we should make two remarks. First of all, Rezk [41] makes a general computation for any $\left(E_{n}\right)^{0}$-cohomology element; we only give here the simplified formula needed for our purposes. On the power operations, we also only use a simplified case, and simplify notation for that purpose. In our notation, $\alpha_{i}$ are the three solutions to the equation

$$
\left([2]_{F} \alpha\right) / \alpha=0
$$

in some integral extension of the ring $\left(E_{2}\right)^{0}$. For $z \in\left(E_{2}\right)^{0}(X)$ for a space $X$, the Ando power operation is then

$$
\psi_{\alpha_{i}}(z) \in\left(E_{2}\right)^{0}\left[\alpha_{i}\right]
$$

which is the image by $x \mapsto \alpha_{i}$ of the element of

$$
\left(E_{2}\right)^{0}(X)[[x]] /[2]_{F}(x)=\left(E_{2}\right)^{0}(X \times B \mathbb{Z} / 2)
$$

which is given by the composition

$$
X \times B \mathbb{Z} / 2 \rightarrow E \mathbb{Z} / 2 \times_{\mathbb{Z} / 2} X^{2} \rightarrow E \mathbb{Z} / 2 \rtimes_{\mathbb{Z} / 2}\left(E_{2}\right)^{2} \rightarrow E_{2} .
$$

Here the first map is the diagonal, the middle map is induced by $z: X \rightarrow E_{2}$, the last map is the power operation in $E_{2}$ given by the fact that $E_{2}$ is an $E_{\infty}$-ring spectrum. Similarly, the operation $\psi_{2}(z) \in\left(E_{2}\right)^{0}(X)$ is defined as the image of $x \mapsto \alpha_{1}, y \mapsto \alpha_{2}$ of the element of

$$
\left(E_{2}\right)^{0}[[x, y]] /\left([2]_{F}(x),[2]_{F}(y)\right)=\left(E_{2}\right)^{0}(X \times B \mathbb{Z} / 2 \times B \mathbb{Z} / 2)
$$

which is given by the composition

$$
\begin{array}{r}
X \times B(\mathbb{Z} / 2 \times \mathbb{Z} / 2) \rightarrow E(\mathbb{Z} / 2 \times \mathbb{Z} / 2) \times_{\mathbb{Z} / 2 \times \mathbb{Z} / 2} X^{4} \\
\rightarrow E(\mathbb{Z} / 2 \times \mathbb{Z} / 2) \rtimes_{\mathbb{Z} / 2 \times \mathbb{Z} / 2}\left(E_{2}\right)^{4} \rightarrow E_{2}
\end{array}
$$

where the maps are analogous as above.

For our purposes, what matters is that for the periodicity element $u \in$ $\left(\widetilde{E_{2}}\right)^{0} S^{2}$, one has

$$
\psi_{\alpha_{i}}(u)=u\left(u+{ }_{F} \alpha_{i}\right),
$$




$$
\psi_{2}(u)=u \prod_{i=1}^{3}\left(u+{ }_{F} \alpha_{i}\right)
$$

while also $u^{2}=0$. Therefore, also using the fact that the Ando operations give a homomorphism of rings, for the generator $u_{k} \in\left(\widetilde{E_{2}}\right)^{0} S^{2 k}$, we get

$$
\begin{gathered}
\sum_{i=1}^{3} \psi_{\alpha_{i}}(u)=u_{k}\left(\left(\alpha_{1}\right)^{k}+\left(\alpha_{2}\right)^{k}+\left(\alpha_{3}\right)^{k}\right), \\
\psi_{2}(u)=u_{k}\left(\alpha_{1} \alpha_{2} \alpha_{3}\right)^{k} .
\end{gathered}
$$

These elements are in $\left(E_{2}\right)^{0}\left(S^{2 k}\right)$. To calculate them, note that

$$
[2]_{F}(x)=2 x+{ }_{F} a x^{2}+{ }_{F} x^{4}=\left(x-\alpha_{1}\right)\left(x-\alpha_{2}\right)\left(x-\alpha_{3}\right)\left(1+K_{1} x+\ldots\right)
$$

(neglecting $u$ and recalling that $\left(E_{2}\right)^{0}=W_{2}[[a]]$ with an element $a$ of dimension 0 - this is not the element $a$ which occurs in the $\mathbb{Z} / 2$-equivariant Steenrod algebra), so

$$
\begin{gathered}
\alpha_{1} \alpha_{2} \alpha_{3}=-2, \\
\alpha_{1} \alpha_{2}+\alpha_{1} \alpha_{3}+\alpha_{2} \alpha_{3}=a+2 K_{1} .
\end{gathered}
$$

We also know

$$
\alpha_{1}+\alpha_{2}+\alpha_{3} \text { is divisible by } 2,
$$

because otherwise (5.23) would not be integral. ¿From these equations, setting

$$
s_{k}=\left(\alpha_{1}\right)^{k}+\left(\alpha_{2}\right)^{k}+\left(\alpha_{3}\right)^{k}
$$

and using Newton's formula, one gets

$$
\begin{gathered}
2 \mid s_{1}=2 a, s_{2} \in 2 J, s_{3} \in 2 J-6, \\
s_{k} \in 2 J \text { for } k>3
\end{gathered}
$$

where $J$ is the ideal in $\left(E_{2}\right)^{0}$ generated by 2 and $a$. Note that only the summand -6 of $s_{3}$ is not in $2 J$. From this, using (5.23), we get the following:

The only non-zero homotopy group of the fiber $F$ of the map $\phi$ of (5.22) in dimension $\geq 3$ is in dimension 5 .

Now $g l_{1}$ is a (topological) right adjoint functor from the homotopy category of spectra to the homotopy category of connected spectra, so for a 
finite group $G$ acting on a spectrum $E, g l_{1}\left(E^{h G}\right)$ is the connective cover of $\left(g l_{1}(E)\right)^{h G}$ where $E^{h G}$ denotes the homotopy fixed point spectrum of $E$ under the $G$-action. Therefore, the above assertion gives

For any action of the group $\mathbb{Z} / 2$ on $E_{2}$, the only non-zero homotopy groups of the fiber $F_{\mathbb{Z} / 2}$ of the map $\phi^{h \mathbb{Z} / 2}: g l_{1}\left(\left(E_{2}\right)^{h \mathbb{Z} / 2}\right) \rightarrow\left(E_{2}\right)^{h \mathbb{Z} / 2}$ in dimensions $\geq 3$ are in dimensions $\leq 5$. Moreover, the homotopy groups in dimensions 3 , 4 are $\mathbb{Z} / 2$-modules.

Now the theory of May, Quinn and Ray [44] shows that for a map of spectra

$$
s p \rightarrow g l_{1}(S)
$$

(where $s p$ is some connective spectrum), and an $E_{\infty}$-ring spectrum $E$, an $E_{\infty}$-orientation from the corresponding Thom spectrum $M s p$ to $E$

$$
M s p \rightarrow E
$$

exists if and only if the composition

$$
s p \rightarrow g l_{1}(S) \rightarrow g l_{1}(E)
$$

vanishes where the second map is induced by the unit of $E$. For example, this can be applied with $s p=\operatorname{Spin}, E=\left(E_{2}\right)^{h \mathbb{Z} / 2}$ for the finite group $\mathbb{Z} / 2$ acting on $E_{2}$. But recall that the only non-zero homotopy group of Spin of dimension $<7$ is $\mathbb{Z}$ in dimension 3 ; therefore, by the above assertion, the map (5.24) must factor through the map

$$
\lambda: \operatorname{spin} \rightarrow \Sigma^{3} H \mathbb{Z} / 2
$$

which induces an onto map in $\pi_{3}$. Therefore, the obstruction vanishes when pulled back to the fiber $s p$ of $\lambda$. However, by general arguments of cobordism theory it follows that the corresponding Thom spectrum $M s p$ classifies precisely Spin-manifolds with $w_{4}=0$. Therefore, we have proved in particular that all such manifolds are $\left(E_{2}\right)^{\mathbb{Z} / 2}$-orientable.

\section{Physical interpretation: The connection between elliptic cohomology and $M$-theory}

In this section, we gather our evidence that elliptic cohomology is very closely and fundamentally tied to $M$-theory. This includes the evidence from the 
previous sections, as well as other clues. We only outline the ideas as we plan to investigate the proposals in this section in more detail and more carefully elsewhere [48]. We continue to work with a 10-dimensional compact Spinmanifold $X$ and put $Y=X \times S^{1}$. Note that elliptic cohomology of $X$ and $Y$ are related by the Künneth theorem: $E^{*}(Y)=E^{*}(X) \otimes_{E^{*}} E^{*}\left(S^{1}\right)$, so

$$
E^{n}(Y)=E^{n}(X) \oplus E^{n-1}(X)
$$

\subsection{The twisting}

Now let us first discuss twisting. In the previous sections, we focused on the untwisted case, but let us now briefly consider the case when the background is twisted by an NS 3 -form $H$-field. In that case, as noted in [1], Section 11, in the IIA part of the discussion, one must replace $K$-theory by twisted $K$-theory, which was done in [24]. However, Douglas [49] remarks that twistings of $K$-theory determine topological modular forms: more precisely, twistings of $K$-theory are classified by the space $B G L_{1}(K)$ and we have a map $B G L_{1}(K) \rightarrow t m f$. Therefore, we see that twistings of $K$-theory are encoded in $\operatorname{tm} f$, which further maps into $E$. Hence, while in twisted $K$-theory we must alter the theory with each $H$-field, elliptic cohomology unifies all of these twisted cases in one theory. An explanation of this phenomenon may again lie in the connection between elliptic cohomology and 2-vector bundles, as discussed in [47], [50].

While $K$-theory twistings give rise to actual topological modular forms, we have argued that complex-oriented elliptic cohomology seems to play a more basic role in the present case. This hints that more "twisting" should be allowed. Presumably, this should be the twisting by the field strength $H_{7}$, which is the field strength associated with the NS5-brane, in a dual way to the situation where $H_{3}$ is associated with the fundamental string $F 1$. The lift to M-theory of the NS branes leads to the M-branes. The fundamental string expands in one dimension along the M-theory circle to become the M2-brane, whereas the NS5-brane lifts to M5 brane, thus maintaing the same worldvolume dimension. The reason for this difference in codimension in the lifting is a consequence of the dimensions of the relevant forms. In 10 dimensions, the NS field strengths have dimensions three and seven, while in eleven dimensions, the fields have dimensions four and seven. 
The main result of [1] shows, in the case $Y=X \times S^{1}$, a match between the partition function of $M$-theory calculated from the $G_{4}$-field, which is the field strength assoicated with the $M 2$-brane, and the IIA-partition function, which is calculated from the fundamental string RR sector. When increasing the coupling in IIA, we get $M$-theory compactified on $X \times S^{1}$, and in this duality, the fundamental string acquires another dimension, and becomes identified with the $M 2$-brane. This again suggests that the elliptic refinement of the partition function which we proposed reflects in some way interaction between the $M 2$ and $M 5$-brane. Note that the $M 5$-brane is an object in $M$-theory, with electro-magnetically dual coupling to the field strength $G_{4}$. In IIA, this object loses one dimension, and becomes the $D 4$-brane. However, using the strong-weak duality, we may also, as above, identify M5 with a 6-dimensional object, namely the $N S 5$-brane in IIA which couples magnetically to the NS charge. From this point of view, the behaviors of $M 5$ and $M 2$ are symmetrical.

If we denote the worldvolume of the NS5-brane by $W$, then the fundamental class $\kappa \in H_{6}(W)$ must satisfy $S q^{3}(\kappa)=0 \in H_{3}(X, \mathbb{Z})$, i.e. $\kappa$ must lift to the $K$-theory homology of $X$. This is also $d^{3}$ in the $E(2)$-homology AHSS of $X$. However, similarly as in Section 5.1 above, the next differential $d^{5}$ lands in homological dimension 1 and hence is excluded, so $\kappa$ lifts to a class in $E(2)_{6}(X)$. Now by multiplying by the elliptic cohomology periodicity element $v_{2}^{-1}$, which is in dimension -6 , we get an element of $E(2)_{0}(X)$.

It is worth commenting that 6 is the only dimension of a world volume $\leq 10$ which can be shifted to 0 by inverting the element $v_{2}$. Note that, unlike in $K$-theory, the Bott element $v_{1}$ is not inverted in elliptic cohomology: this may be singling out the 5-brane as the object whose interactions give the main part of the $M$-theoretical correction to the IIA partition function. In $M$-theory, the $M 5$-brane couples magnetically to the field strength $G_{4}$. In [1], Section 7.2, arguments are given pointing out the naturality of a choice of coordinates under which $G_{4}$ gives the main contribution to their partition function (the approximation with which we are working here). ¿From another point of view, in type II string theory, RR $D$-branes of lower dimension can be generated from higher dimension by tachyonic condensation. This involves the Gysin isomorphism, i.e. the Bott element. In $M$ theory, as not all even dimensions of branes are allowed (specifically, we cannot reverse the process and for example turn a 2 -brane into a 4 -brane in $M$-theory). This, 
again, seems related to the non-invertibility of the element $v_{1}$ in elliptic cohomology.

But what might be the twisting with respect to the field strength $H_{7}$ ? For this, note that $v_{2} \in M U_{*}$ is represented by a complex manifold, namely the Milnor manifold whose Segre characteristic number is 2. However, an M5-brane, while it must be orientable, may not be a complex manifold. Therefore, it is reasonable to propose that inclusion of such non-complex M5-branes will introduce a new twisting.

Elliptic cohomology has the formal group law of an elliptic curve. Hence one should be able to see the group (or possibly subgroups of) $S L(2, \mathbb{Z}$ ). Since the modular parameters that appear in M-theory and string theory are usually of the form (e.g. $[55,56])$

$$
\tau=(\text { field })+i(\text { volume modulus })
$$

it seems reasonable to propose the moduli in the form

$$
\begin{aligned}
& \tau_{2}=\left\langle B_{2},\left[\Sigma_{2}\right]\right\rangle+\operatorname{ivol}\left(\Sigma_{2}\right) \\
& \tau_{6}=\left\langle B_{6},\left[\Sigma_{6}\right]\right\rangle+\operatorname{ivol}\left(\Sigma_{6}\right)
\end{aligned}
$$

where $\langle$,$\rangle is the Kronecker product, [ ] is the fundamental class, \Sigma_{2}$ and $\Sigma_{6}$ are two- and six-cycles that can correspond to $F 1$ and $N S 5$ respectively. The modular parameter $\tau_{6}$ should be related to the map (4.1) by an equation which we can schematically write as

$$
q=e^{2 \pi i \tau_{6}} .
$$

Note however that in order to make physical predictions from this, we would need a more precise normalization of coordinates to predict the exact choice of group of modular transformations (see also the comments on elliptic spectra in Appendix B), as well as a formula for the M5-brane charge (see next Section).

\subsection{M2 and M5-branes}

It remains to give an interpretation of $v_{2}^{-1} v_{1}^{3}$. One possible suggestion in the corresponding M-theory picture is that the $M 2$ and the $M 5$ coexist and that 
the membrane modulus is not inverted. The first point can be understood from

M-theory as the general statement that if a soliton spectrum contains the $M 5$-brane then it automatically contains the $M 2$-brane. This can be understood by the Hanany-Witten effect that implies that an M2-brane is created when two fivebranes cross. Alternatively, an M2-brane appears from dielectric $M 5$-branes in the limit when the 3 -cycle shrinks to zero.

Let us review briefly the intersections and bound states of M-branes. M2 and M5 can consistently coexist, in compatability with the gravitational anomaly cancellation. They obey a Dirac quantization condition $e g=2 \pi n G_{N}$ where $e$ is the M2 charge and $g$ is the M5 charge and $G_{N}$ is the eleven-dimensional Newton constant. The presence of M2 and M5 modify the equation of motion to 1011

$$
d * G_{4}=G_{4} \wedge G_{4}+g f_{3} \wedge J_{5}+e J_{8}+\frac{2 \pi G_{N}}{g} X_{8}
$$

where $J_{5}$ is the current (poincaré dual) of M5, and $J_{8}$ that of M2. $X_{8}$ is the 8-polynomial associated with the gravitaional anomaly, and $f_{3}$ is the $M 5$ worldvolume gauge field. From supergravity, the allowed supersymmetric intersections (see e.g. [52] for a review) include: ${ }^{12} M 2 \cap M 2(0), M 2 \cap M 5(1)$, $M 5 \cap M 5(1)$, and $M 5 \cap M 5(3)$. The second is especially interesting because it is the intersection over a string and is one of the building blocks for brane-intersections. The reduction of the intersection $M 2 \cap M 5(1)$ along M2 leads to F1 ending on NS5, and leads to D2 ending on D4 if the reduction is along one of the coordinates of M5. There are many possible D-brane bound states in type II string theory. One way they arise is by placing D-branes in constant background B-field. The worldvolume coordinates of the Dp-brane become noncommutative $(=\mathrm{NC})$ along the directions of the non-vanishing B-field. If B is spacelike, one can define a decoupling limit of NCYM, i.e. a $\mathrm{NC}$ field theory. If $\mathrm{B}$ is timelike, one gets noncommutative open string theory (NCOS). In principle, any bound state in type IIA should have a lift to M-theory, and the analogue situation is M-branes in (constant) back-

\footnotetext{
${ }^{10}$ Such a modification has been discussed in [51] using Chern-kernels.

${ }^{11}$ A more careful analysis would perhaps involve more refined treatment like CheegerSimons differential characters [22]. As we mentioned earlier, we will investigate the ideas of this section more carefully in [48].

${ }^{12}$ notation: $(p)$ means the intersection is over a p-brane. Note that these are orthogonal intersections.
} 
ground $C$-field. The configuration of M2 branes ending on M5 is the lift of strings ending on D-branes. One can similarly define a decoupled theory, the light Open Membrane (OM) theory [53]. Six-dimensional OM-theory is the high energy limit of 5-dimensional NCYM and NCOS. Compactification of OM-theory on an electric (resp. magnetic) circle leads to NCOS (resp. NCYM). A constant background C-field can be traded for a constant M5 worldvolume field $f_{3}$. This represents a bound state of this M5 with a delocalized M2 along 2 of the 5 spatial directions of M5. Many of the bound states involving different combinations can be related to $(M 2, M 5)$ by a (Lorentz) transformation, and this seems to indicate that the latter is the "basic" bound state.

Perhaps even more importantly for us, however, this suggests that we may use the IIA-side of the [1] calculation to interpret the elliptic refinement, as the $G_{4}$ approach should be exactly dual. The beginning of such argument was seen at the end of the last section. But the IIA-side is easier to work with, as we have at our disposal the usual expansion of the radial excitation modes of a fundamental string. As we already saw, the suggestion about the meaning of the parameter $v_{2}^{-1} v_{1}^{3}$ is that it comes from interaction with a complex oriented $M 5$-brane, and that the partition function should be twisted if the $M 5$-brane is not complex-oriented. Therefore, we should consider what kind of possible bound states between $M 2$ and $M 5$-branes can arise. One striking suggestion is an open $M 2$-brane with boundary on the M5-brane. One feature which could suggest those states is the non-invertibility of $v_{1}$, and hence the element $v_{2}^{-1} v_{1}^{3}$, in elliptic cohomology. However, from the point of view of the $M 5$-brane, it is not clear whether such states would not be anomalous. Also, in the IIA dimensional reduction, we are not seeing any direct role of the open string partition function. It may therefore be that rather than ending on the $M 5$-brane, the $M 2$-brane intersects the $M 5$-brane in a fundamental string, and the elliptic partition function reflects the energy such bound state acquires from the intersection. One might also argue that in analogy to the string theory situation where the open sector requires the existence of the closed sector, the open membrane requires the existence of the closed membrane. However, the open states seem to be only needed in this setting to imply the existence of the branes, and they do not enter the calculations of the partition function. ${ }^{13}$

\footnotetext{
${ }^{13} \operatorname{In}[1,23,24]$, the branes are not directly used in calculating the partition function, and in discussing them, we are assuming further that one can include them and use them
} 
We therefore conjecture that the elliptic refinement of the IIA- (and alternately the M-theory $G_{4^{-}}$) partition function picks up states arising from intersection of an $M 2$-brane with an $M 5$-brane, which could be $H_{7}$-twisted if the M5-brane is not complex-oriented. In the present (untwisted) case, there is an anomaly of these states when $w_{4} \neq 0$. On the IIA side for $Y=X \times S^{1}$ (the case considered here), the intersection is the (end of the) IIA fundamental string. The elliptic partition function which we constructed, after suitable normalization and computation of the 5-brane charge should compute the the $M 2-M 5$-intersecting state correction to the $G_{4}$ M-theory partition function.

In section 5.2 we proved that orientability with respect to $E O(2)$ is equivalent to vanishing of $w_{4}$. We would like to point out the relevance of this to the M-theory backgrounds as well as to the $M 5$ anomaly. In [19], Witten showed that $G_{4} / 2 \pi$ is quantized as $w_{4} / 2 \bmod \mathbb{Z}$. The the condition $w_{4}=0$ implies that there are no half-integral fluxes, which is the case for the relevant $\mathbb{Z}_{2}$-orbifolds (and orientifolds). Later in [20], Witten showed that $w_{4}$ also shows up as (part of the) mod 2 index and, consequently, the anomaly for the $M 5$-brane. We will revisit this in [48] and study possible relation between 1/2-integral fluxes and twisting by $H_{7}$ in this context.

\subsection{Closing remarks}

There is also a purely mathematical side of these phenomena: In [54], a model of elliptic cohomology was proposed based on $\mathcal{E}$-equivariant stringy bundles over an elliptic curve $\mathcal{E}$. A stringy bundle is a variant of what $[45,31]$ call an elliptic object, i.e. a "conformal field theory indexed by $\mathcal{E}$ ". The authors of [54] could not figure out what was the role of the elliptic curve $\mathcal{E}$ in the "spacetime" part of the theory. But the present context suggests that this may be perhaps interpreted as an intersection between an M2-brane and an $M 5$-brane, and that the roles of these $M$-branes should be further investigated to enhance geometric interpretation of elliptic cohomology. It should be emphasized that in [54], there was no object which would play the role of the spacetime manifold $X$, as the mathematical story is concerned with abstract CFT only, and not superstring theory of a given type. However, the present observations may suggest that even there, interactions of

for the spacetime result. 
the $M 2$-brane and $M 5$-brane may play a role.

The physical interpretation in this section deserves to be studied more carefully and in more detail, in particular on the topics of normalization and identification of modular group, as well as an $M 5$-brane charge formula. We hope to achieve this in [48].

M-theory continues to prove how rich it is both physically and mathematically. We hope that elliptic cohomology and Morava K-theory could be "derived" in the future from M-theory, at the level of partition functions, in the sense of [1] for K-theory and help in completing the derivation initiated in [24] for twisted K-theory.

\section{Acknowledgements}

H. S. would like to thank V. Mathai for useful discussions on [1]. He also thanks the Michigan Center for Theoretical Physics for hospitality, during which this project was started. The authors would like to thank Matthew Ando, Mike Hopkins and Charles Rezk for discussions, especially on their theory of $t m f$-orientability.

The first author's research was supported by NSF grant DMS 0305853. The second author's research was supported by the Australian Research Council. 


\section{A Appendix: Orientability}

In this appendix, we collect some definitions and basic properties of orientation [57]. Let us start with the simplest case. The orientation of $\mathbb{R}^{n}$ can be defined homologically as one of the generators of the group $\mathbb{Z}=H_{n}\left(\hat{\mathbb{R}}^{n}, \mathbb{Z}\right)=H^{n}\left(\hat{\mathbb{R}}^{n}, \mathbb{Z}\right)$ where $\hat{\mathbb{R}}^{n}=S^{n}$ is the one-point compactification.

If $\mathrm{X}$ is a closed connected manifold with $H_{n}(X, \mathbb{Z})=\mathbb{Z}$ then every generator $[X]$ of $H_{n}(X, \mathbb{Z})$ can be considered as an orientation of $X$.

A $\mathbb{R}^{n}$-bundle $\xi$ over a connected manifold is orientable if $H^{n}(T \xi, \mathbb{Z})=\mathbb{Z}$, where $T \xi$ is the Thom space of $\xi$. An orientation of $\xi$ is a generator of $H^{n}(T \xi, \mathbb{Z})$.

For an arbitrary cohomology theory $E$, an $E$-orientation of a closed manifold $X^{n}$ is an element $[X] \in E_{n}\left(X^{n}\right)$.

Examples of orientation:

1. $H \mathbb{Z}$-orientability: A vector bundle $\xi$ is $H \mathbb{Z}$-orientable if and only if its structure group can be reduced to $S O$. This holds if and only if $w_{1}(\xi)=0$. By using the Thom isomorphism $w_{1}(\xi)=\phi^{-1} S q^{1} u_{\xi}$, this is equivalent to $S q^{1}(u)=0$, where $u=u_{\xi} \in H^{n}\left(T \xi, \mathbb{Z}_{2}\right)$.

2. KO-orientability: A vector bundle $\xi$ is $K O$-orientable if and only if it admits a spin structure, i.e. if and only if $w_{1}(\xi)=0=w_{2}(\xi)$.

3. K-orientability: A vector bundle $\xi$ is $K$-orientable if and only if it admits a $\operatorname{spin}^{c}$ structure, i.e. if and only if $w_{1}(\xi)=0=\beta w_{2}(\xi)$. Note that every $K O$-orientable vector bundle is also $K$-orientable.

4. E-orientability: Let $X$ be a topological manifold. An element $[X, \partial X] \in$ $E_{n}(X, \partial X)$ is an $E$-orientation if

$$
\mathcal{E}_{*}^{m, U}[X, \partial X]= \pm s_{n}
$$

for every $m \in X$ and every disk neighborhood of $m$. Here $\mathcal{E}^{m, U}: X \rightarrow$ $S^{n}$ is the map that collapses the complement of $U . s_{n} \in E_{n}\left(S^{n}, *\right)$ is the image of $1 \in \pi_{0}(E)$ under the isomorphism

$$
\pi_{0}(E)=\tilde{E}_{0}\left(S^{0}\right) \cong \tilde{E}_{n}\left(S^{n}\right)=E_{n}\left(S^{n}, *\right)
$$


and is a canonical orientation of the sphere $S^{n}$. A closed manifold is $E$-orientable iff its stable normal bundle is $E$-orientable.

Oriented objects have a lot of good properties:

1. There is a Thom-Dold isomorphism $\phi: E^{i}(X) \rightarrow \tilde{E}^{i+n}(T \xi)$ for every $E$-oriented $\mathbb{R}^{n}$-bundle $\xi$ over X.

2. There is Poincaré duality: $E^{i}(X) \rightarrow E_{n-i}(X, \partial X)$ for every $E$-oriented manifold $X^{n}$.

3. One can generalize the classical Chen classes and develop a theory of characteristic classes taking values in $E^{*}$ provided all complex vector bundles are $E$-orientable. Those are called Chern-Dold classes.

4. They provide integral invariants. Start with any $D$-orientable manifold $X^{n}$, having $D$ - and $E$ - orientations $[X]_{D}$ and $[X]_{E}$, respectively. Let $\tau$ : $D \rightarrow E$ be a ring morphism of ring spectra. Let $\xi$ be any $D$-orientable (and hence $E$-orientable) object over $X$, and let $u_{D}$ (rep. $u_{E}$ ) be a $D$ - (reps. $E$-) orientation of $\xi$. Integrality phenomena arise because of incompatability of the orientations, i.e. $\tau\left(u_{D}\right) \neq u_{E}$. The orientation $u_{E}$ gives rise to the Thom-Dold isomorphism $\phi_{E}: E^{*}(X) \rightarrow \tilde{E}^{*}(T \xi)$. Set

$$
R(\xi)=R_{u_{D}, u_{E}}(\xi):=\phi_{E}^{-1} \tau\left(u_{D}\right) \in E^{0}(X)
$$

The orientations $[X]_{D}$ and $[X]_{E}$ determine orientations $u_{D}(\nu)$ and $u_{E}(\nu)$ of the stable normal bundle of $X$. Then, one can write equate Kronecker pairings using the two different orientations

$$
\left\langle\tau(x) R(\nu),[X]_{E}\right\rangle=\tau\left\langle x,[X]_{D}\right\rangle
$$

The integrality theorem says that the element $\left\langle\tau(x) R(\nu),[X]_{E}\right\rangle$ of the group $\pi_{n-k}(E)$ belongs to the subgroup $\operatorname{Im}\left\{\tau_{*}: \pi_{n-k}(D) \rightarrow \pi_{n-k}(E)\right\}$.

5. Gysin homomorphism: Let $F: X^{m} \rightarrow Y^{n}$ be a map of closed manifolds. If both $X, Y$ are E-oriented, then one can define the Gysin homomorphisms:

$$
f^{!}: F^{i}(X) \rightarrow F^{n-m+i}(Y), \quad f_{!}: F_{i}(X) \rightarrow F_{n-m+i}(Y)
$$

to be the compositions

$$
\begin{aligned}
f^{!}: F^{i}(X) & \cong F_{m-i}(X) \stackrel{f_{*}}{\longrightarrow} F_{m-i}(Y) \cong F^{n-m+i}(N), \quad f^{!}=P_{[Y]}^{-1} f_{*} P_{[X]} \\
f_{!}: F_{i}(Y) & \cong F^{n-i}(X) \stackrel{f_{*}}{\longrightarrow} F^{n-i}(X) \cong F_{m-n+i}(Y), \quad f_{!}=P_{[X]} f^{*} P_{[Y]}^{-1}
\end{aligned}
$$


If $Y$ and/or $X$ are not $E$-orientable but the difference of normal bundles $\nu_{Y}-f^{*} \nu_{X}$ is, then one can still define similar maps.

In particular, if $m=n$, the manifolds are $H \mathbb{Z}$-oriented and the map has degree \pm 1 then $Y$ is $E$-orientable if $X$ is.

The obstruction to orientability is given by some invariants called the Postnikov invariants. For our purposes, an object is $E$-orientable iff $0 \in \kappa_{n}$ for all $n$, where $\kappa_{n} \in H^{n+1}\left(E_{(n+1)} ; \pi_{n}(E)\right)$ is the $n$-th Postnikov invariant of $E$.

\section{B Appendix: Formal group laws, Milnor primi- tives and a menagerie of generalized cohomol- ogy theories}

We recall here very briefly the story of formal group laws (FGL's), and how they are relevant to complex-oriented generalized cohomology. The reader may refer to Appendix 3 of [34] for more detailed information. What we mean by a (1-dimensional, commutative) formal group law over a (super)commutative ring $R$ is a series

$$
F(x, y)=x+_{F} y=\sum a_{i j} x^{i} y^{j} \in R[[x, y]]
$$

which satisfies

$$
\begin{aligned}
x+{ }_{F} 0 & =x, \\
x+{ }_{F} y & =y+{ }_{F} x, \\
\left(x+{ }_{F} y\right)+_{F} z & =x+{ }_{F}\left(y+{ }_{F} z\right) .
\end{aligned}
$$

The significance in homotopy theory is as follows: we call a ring-valued generalized cohomology theory $E$ complex-oriented if the canonical complex line bundle $\gamma$ over $\mathbb{C} P^{\infty}$ is $E$-oriented, i.e. satisfies an $E$-Thom isomorphism. A choice of such isomorphism is called a complex orientation of $E$. It then follows that every complex bundle is E-oriented. Moreover, the complex orientation determines a Chern classes of complex bundles precisely analogously as in the case of ordinary cohomology. The way FGL's enter the picture is that there exists a unique FGL over the ring $E_{*}$ (depending only 
on $E$ and complex orientation) such that for complex line bundles $L, M$ over a space $X$,

$$
c_{1}(L \otimes M)=c_{1}(L)+{ }_{F} c_{1}(M)
$$

One should note that if we change the complex orientation on $E$, the formal group law gets replaced by a strongly isomorphic FGL $G$, which means that there exists a formal series $f(x)=x+a_{2} x^{2}+a_{3} x^{3}+\ldots \in E_{*}[[x]]$ such that

$$
f(x)+{ }_{G} f(y)=f\left(x+{ }_{F} y\right) .
$$

The formal group law up to strong isomorphism is a powerful invariant of a complex-oriented generalized cohomology theory. The FGL of ordinary cohomology is additive, i.e. $x+{ }_{F} y=x+y$, and the FGL of $K$-theory is multiplicative, i.e. $x+{ }_{F} y=x+y+v_{1} x y$ where $v_{1}$ is the Bott element. It should be noted that over a $\mathbb{Q}$-algebra, all FGL's are isomorphic, just as all rational generalized cohomology theories are equivalent: this is a manifestation of the fact that homotopy theory is, very much, "the science of torsion". There exists a universal formal group law, i.e. a commutative ring $L$ with a formal group law $F$ such that for any (super)-commutative ring $R$ and any FGL $G$ on $R$ there is a unique homomorphism of rings $L \rightarrow R$ which carries $F$ to $G$. This ring $L$ was discovered by Lazard. Quillen discovered that, miraculously, $L$ is isomorphic to the ring of coefficients of $M U$, complex cobordism, which is in some sense the universal complex-oriented generalized cohomology theory!

The story goes onwards from there. If we localize at a prime $p$ (in this paper, the relevant case is $p=2$ ), then $M U$ breaks up as a direct sum of certain theories called BP after Brown-Peterson [32]. There is a corresponding notion of $p$-typical FGL, which however we shall not need to consider. In any case, the coefficients of $B P$ are

$$
B P_{*}=\mathbb{Z}\left[v_{1}, v_{2}, v_{3}, \ldots\right], \quad \operatorname{dim}\left(v_{n}\right)=2 p^{n}-2 .
$$

The formal group law on $B P_{*}$ (the universal $p$-typical FGL) was also first observed in algebra and is due to Cartier. By work of Baas-Sullivan (with substantial recent improvements and simplifications), it is possible to create generalized cohomology theories at will by killing regular sequences in the ring $B P_{*}$ and/or inverting elements in its coefficient ring. This leads to a "menagerie of generalized cohomology theories". Some of the important 
theories are the Johnson-Wilson theory $B P\langle n\rangle$ with coefficients $\mathbb{Z}\left[v_{1}, \ldots, v_{n}\right]$ and corresponding theory with $v_{n}$ inverted which is the Landweber theory denoted by $E(n)$ (hence, $E(n)_{*}=\mathbb{Z}\left[v_{1}, \ldots, v_{n}, v_{n}^{-1}\right]$ ). One may also kill all of the lower $v_{n}$ 's to get the integral Morava $K$-theory $\tilde{K}(n)$ with coefficients $\mathbb{Z}\left[v_{n}, v_{n}^{-1}\right]$, or kill also $p$ to get the Morava $K$-theory $K(n)$ with coefficients $\mathbb{Z} / p\left[v_{n}, v_{n}^{-1}\right]$. There are also connective versions of the Morava theories $\tilde{k}(n)$, $k(n)$ with coefficients $\mathbb{Z}\left[v_{n}\right], \mathbb{Z} / p\left[v_{n}\right]$, respectively.

Some of these theories have notable formal group laws. For example, the formal group law on $K(n)_{*}$ has height $n$, which means that

$$
[p]_{F} x=\underbrace{x++_{F} \ldots++_{F} x}_{p \text { times }}=v_{n} x^{p^{n}} .
$$

Studying isomorphisms of this FGL led Lubin and Tate [38] to the discovery of the Lubin-Tate FGL on the ring $W_{n}\left[\left[a_{1}, \ldots, a_{n-1}\right]\right]\left[u, u^{-1}\right]$ where $W_{n}$ is the ring of Witt vectors, i.e. integers of an Eisenstein extension of order $p^{n}$ of the field of $p$-adic numbers $\mathbb{Q}_{p}$. The Lubin-Tate coefficient ring turns out to be a completed sum of copies of $E(n)_{*}$, and one can consider a generalized cohomology theory with the Lubin-Tate coefficient ring, which is essentially a completed sum of copies of $E(n)$. However, the reason Lubin-Tate laws were invented has nothing to do with homotopy theory; rather, they are needed for local class field theory (algebraic number theory).

For $n=2$, one can also consider cohomology theories whose formal group laws are elliptic, i.e. are obtained by Taylor expansion of the group law on an elliptic curve over some commutative ring. Such theories are called complexoriented elliptic cohomology theories. To be more precise about this, Ando, Hopkins and Strickland [37] define a 2-periodic ring spectrum as a generalized cohomology theory $E$ with an orientation of the identical complex line bundle on $\mathbb{C} P^{\infty}$ which, when restricted to $\mathbb{C} P^{1}$, has an inverse in $E_{*}$. If in addition $E_{2 n+1}=0$ for all $n$ (an evenness condition) and there is an elliptic curve $\mathcal{E}$ over $E_{*}$ with an isomorphism of its formal group law with the FGL of $E$, [37] call $E$ an elliptic spectrum. (Note: the word "spectrum" is a term of algebraic topology which means a generalized cohomology theory. The reason for using a separate word is that spectra may be refined to a category which contains rigid point set level information, analogous to point set level maps of spaces. Cohomology theories, on the other hand, see only maps "up to homotopy". The rigid level is necessary for certain more complicated 
constructions, such as (co)simplicial realizations.)

Among the examples given in [37] is the elliptic spectrum

$$
E_{*}=\mathbb{Z}\left[a_{1}, a_{2}, a_{3}, a_{4}, a_{6}\right]\left[u, u^{-1}\right]
$$

associated with the Weierstrass curve which we write in the form

$$
y^{2}+a_{1} u x y+a_{3} u^{3} y=x^{3}+a_{2} u^{2} x^{2}+a_{4} u^{4} x+a_{6} u^{6} .
$$

Here we put $\operatorname{dim}(x)=4, \operatorname{dim}(y)=6$. The map (4.1) for this spectrum can be constructed using the so called Tate parametrization. Letting $\sigma_{k}(n)=$

$$
\begin{aligned}
& \sum_{d \mid n} d^{k}, \alpha_{k}=\sum_{n>0} \sigma_{k}(n) q^{n}, \text { one can define (4.1) by } \\
& \quad a_{1}=1, a_{3}=0, a_{2}=0, a_{4}=-5 \alpha_{3}, a_{6}=-\left(5 \alpha_{3}+7 \alpha_{5}\right) / 12 .
\end{aligned}
$$

(The coefficients of $a_{6}$ turn out to be integers.)

On the other hand, the Lubin-Tate spectrum

$$
E_{*}=W_{2}[[a]]\left[u, u^{-1}\right]
$$

is also an elliptic spectrum, its corresponding elliptic curve being

$$
y^{2}+a u x y+u^{3} y=x^{3}
$$

(see [59]). To construct the map (4.1) in this case (where the target is $K$-theory with coefficients in $W_{2}$ ), the Lubin-Tate FGL has

$$
[2]_{F} x=(2 x)+{ }_{F}\left(a u x^{2}\right)+{ }_{F}\left(u^{3} x^{4}\right)
$$

and Lubin-Tate theory implies that over a ring where $a u$ is invertible, this is isomorphic to the multiplicative FGL. Thus, if we define (4.1) by

$$
a u=v_{1}, u=v_{1} q^{-1}
$$

or equivalently

$$
a=q, u=v_{1} q^{-1},
$$

we get the correct map after composition with the automorphism of $(K \otimes$ $\left.W_{2}\right)[[q]]\left[q^{-1}\right]$ which sends the FGL (B.11) to the multiplicative FGL of $K$ theory. We see that (B.10) is a Weierstrass curve with

$$
a_{1}=a, a_{3}=1, a_{2}=a_{4}=a_{6}=0,
$$


but the map (4.1) we constructed for this theory is however not the same as the map coming from the Tate parametrization, thus further confirming our observation about non-uniqueness of coordinates. Another disadvantage of the theory (B.9) is that the character map requires us to use $K \otimes W_{2}$ and hence the Chern character involves crystalline cohomology.

Regarding maps between the generalized cohomology theories mentioned, a pretty good guideline is usually what maps there are on coefficient rings (without renaming elements). Thus, there are maps from $B P$ to all the theories mentioned, and reductions from $B P\langle n\rangle$ to $\tilde{k}_{i}$ to $k_{i}, 1 \leq i \leq n$. There are also maps $B P\langle n\rangle \rightarrow E(n)$ and $\tilde{k}_{n} \rightarrow \tilde{K}(n), k(n) \rightarrow K(n)$. There are no maps between the $K(n)$ 's for different $n$. However, there is an elliptic curve called the Tate curve, which can be, formally at least, described as " $\mathbb{G}_{n} /\left(q^{\mathbb{Z}}\right)$ " where $\mathbb{G}_{m}$ is the multiplicative group. This Tate curve has coefficients (domain of definition) $\mathbb{Z}[[q]]\left[q^{-1}\right]$ and its formal group law therefore is multiplicative. We therefore have character maps such as (4.1) from elliptic cohomology theories to $K[[q]]\left[q^{-1}\right]$ or its suitable completion.

An important part of stable homotopy story is locality with respect to generalized cohomology theories, in the sense of Bousfield. We say that a spectrum $X$ is acyclic with respect to a generalized cohomology theory $E$ if $E^{*} X=0$. We say that a spectrum $Y$ is $E$-local if $Y^{*} X=0$ whenever $X$ is $E$-acyclic. Bousfield [58] constructed an E-localization map

$$
X \rightarrow L_{E}(X)
$$

which is a universal map (in the homotopy sense) from $X$ to an $E$-local spectrum. One should mention that localization with respect to the Moore spectrum $M \mathbb{Z} / p$ (the cofiber of the degree $p$ map $S^{0} \rightarrow S^{0}$ ) is the right notion of $p$-completion of spectra. For many considerations in homotopy theory, such completion at a prime $p$ is understood throughout without being explicitly mentioned: this is what homotopy theorists mean by working at a prime $p$. Now one distinction between the elliptic cohomology theories mentioned above is behavior with respect to localization. For example, the spectrum $E_{2}$ is $K(2)$-local (recall that $K(2)$ is the second Morava $K$-theory). On the other hand, the spectrum $K[[q]]\left[q^{-1}\right]$ is $K$-local). The spectrum tmf is $E(2)$-local.

In homotopy theory, the story behind complex-oriented cohomology the- 
ories is largely governed by the Milnor primitives. Milnor [33] found that the dual of the Steenrod algebra $A_{*}$ can be simply described as follows (we shall work at $p=2$ ): there is a comultiplication $\psi: H^{*}(X, \mathbb{Z} / 2) \rightarrow$ $H^{*}(X, \mathbb{Z} / 2) \hat{\otimes} A_{*}$ for any space $X$ ( $\hat{\otimes}$ denotes completed tensor product). Now one has for the generator $a \in H^{1}\left(\mathbb{R} P^{\infty}, \mathbb{Z} / 2\right)$,

$$
\psi(a)=\sum a^{2^{i}} \otimes \zeta_{i}
$$

and, in fact, $A_{*}=\mathbb{Z} / 2\left[\zeta_{1}, \zeta_{2}, \ldots\right], \operatorname{dim}\left(\zeta_{i}\right)=2^{i}-1$. So, one can obtain a basis of $A^{*}$ by dualizing monomials in the $\zeta_{i}$ 's. It turns out, however, that the beneficial way of doing this is to dualize the basis

$$
\zeta_{1}^{\epsilon_{1}} \ldots \zeta_{n}^{\epsilon_{n}} \ldots \zeta_{1}^{2 r_{1}} \ldots \zeta_{n}^{2 r_{n}} \ldots
$$

Here $\epsilon_{i} \in\{0,1\} \quad r_{i} \in \mathbb{N}$ and obviously only finitely many of the $\epsilon_{i}$ 's and $r_{i}$ 's are allowed to be non-zero. The reason to write the basis in the awkward way (B.13) is that the dual basis is then of the form

$$
Q_{0}^{\epsilon_{1}} \ldots Q_{n-1}^{\epsilon_{n}} \ldots P^{\left(r_{1}, r_{2}, \ldots\right)}
$$

where the $Q_{i}$ 's form an exterior subalgebra of $A^{*}$. These elements are called the Milnor primitives. The Milnor basis is different from the Cartan-Serre basis, but the Adem relations lead to a recursive conversion formula between both bases. It is not difficult to work out the conversions explicitly in low dimensions, where there are not many elements to consider.

Now the connection with complex-oriented generalized cohomology theories is as follows: Brown-Peterson [32] found that the primary Postnikov invariants (the ones not attached to lower ones) of $B P$ are precisely the Milnor primitives $Q_{n}$, and moreover the invariant $Q_{n}$ attaches precisely the homotopy class $v_{n}$. Since we saw that all of the other theories we wrote down receive maps from $B P$ which preserve names of generators, similar conclusions hold for all of the theories involved, in particular Morava $K$-theories. We therefore conclude that $Q_{n}$ is for example the primary differential of the $K(n)$-AHSS (in homology or cohomology), and its integral lift is the primary differential in the $\tilde{K}(n)$-AHSS, etc. These facts we are using in the present paper.

There are refinements beyond the complex-oriented story which one needs to consider. In particular, one can study real-oriented generalized cohomology theories (see [39]), generalizing orthogonal $\mathrm{KO}$-theory, or more precisely, 
Atiyah's real $K R$-theory. The story is substantially more complicated there, but the basic guideline is that there are real-oriented analogues to all the complex oriented theories considered above. (Caution: the real-oriented analogue of $M U$ is not $M O$ but Landweber's real cobordism $M \mathbb{R}$.) The right way of considering real-oriented generalized cohomology theories is as $\mathbb{Z} / 2$-equivariant theories. This means that homology and cohomology gets doubly indexed, i.e. by $k+\ell \alpha$ where $k, \ell \in \mathbb{Z}$, and $\alpha$ is the sign representation of $\mathbb{Z} / 2$. There is always a forgetful map from a real to the corresponding complex theory, which just sends $\alpha$ to 1 . Now the main point is that complex bundles $\eta$ with a real structure (i.e. isomoprhism $\bar{\eta} \cong \eta$ ) are orientable with respect to real-oriented generalized cohomology theories, and the orientation lies in dimension $n(1+\alpha)$ where $n$ is the dimension of the bundle. The formal group law story is thus repeated from the complex case, but the calculation of coefficients is substantially more difficult (many cases were worked out in [39] and in subsequent papers).

One case which is particularly relevant here is the theory $E O(2)$ which is the fixed point part of the theory $E \mathbb{R}(2)$, the real version of $E(2)$. (Caution: Hopkins-Mahowald [59] use symbols such as $E O(2)$, and in particular $E O_{2}$ in a different meaning than [39], namely to denote fixed point spectra of $E_{2}$ under a larger finite group.) The coefficient ring $E \mathbb{R}(2)_{\star}$ (here the symbol $\star$ means that we are considering the general dimension $k+\ell \alpha$, see [39]) is a $\mathbb{Z}[a] /(2 a)$-module where $a$ has dimension $-\alpha$. The generators of this module are monomials (let us call them admissible) of the form

$$
v_{0}^{\epsilon} v_{1}^{m} v_{2}^{n} \sigma^{2 p}
$$

where $m=\{0,1,2, \ldots\}, n \in \mathbb{Z}, \epsilon=0$ if $m>0$ and $p$ is even or $p$ is divisible by 4 and $\epsilon=1$ otherwise. The only relation on the $\mathbb{Z}[a] /(2 a)$ module generators (B.15) is that those with $\epsilon=1$ are annihilated by $a$. The dimension of each element (B.15) is determined by setting the dimension of $v_{i}$ equal to $\left(2^{i}-1\right)(1+\alpha)(i=0,1,2)$. The dimension of $\sigma$ is $\alpha-1$. The notation for the generators (B.15) comes from a spectral sequence used to calculate $E \mathbb{R}(2)_{\star}$. This notation suggests that the multiplicative structure on $E \mathbb{R}(2)_{\star}$ could be computed simply by multiplying the monomials (B.15). This multiplicative structure is in effect right, if we put $v_{0}^{2}=2 v_{0}([39])$.

To make (B.15) seem a little less exotic, it may be helpful to note that the coefficients $K R_{\star}$ of Atiyah's real $K R$-theory have an analogous description: 
the $\mathbb{Z}[a] /(2 a)$-generators are

$$
v_{0}^{\epsilon} v_{1}^{n} \sigma^{2 p}
$$

where $n \in \mathbb{Z}$, and $\epsilon=0$ if $p$ is even and $\epsilon=1$ if $p$ is odd. One has the relation $v_{0} a=0$ and $v_{0}^{2}=2 v_{0}$.

In this paper, we have considered real-oriented elliptic cohomolgoy $E O(2)$, which can be thought of as the "fixed points" of elliptic cohomology with respect to the formal inverse. However, there is more to the story. Hopkins and Miller [36], see also [37] have constructed an even much more elaborate generalized cohomology theory tmf (which stands for topological modular forms). Roughly speaking, to understand $t m f$, one must study the coordinate changes of the Weierstrass curve. These changes may be encoded in an affine algebraic groupoid, the ring of whose coefficients turns out to coincide with the 0-dimensional part of the coefficients of a cosimplicial elliptic spectrum $\operatorname{tm} f_{*}$; its realization is $\operatorname{tm} f$. The construction of the cosimplicial elliptic spectrum $t m f_{*}$ (in particular making that the cosimplicial structure strict, not just up to homotopy) is the deep part of the theory, and is the subject of the work of [36], much of which is still unpublished. The theory tmf has the striking property that it is $M O\langle 8\rangle$-orientable, i.e. a manifold is $t m f$-orientable if it is Spin and has $p_{1} / 2=0$. This was proved by Ando, Hopkins and Rezk in [40], and a part of their theory was also used in the present paper in Section 5.2 above.

\section{References}

[1] E. Diaconescu, G. Moore and E. Witten, E $E_{8}$ gauge theory, and a derivation of K-Theory from M-Theory, Adv. Theor. Math. Phys. 6 (2003) 1031, [arXiv:hep-th/0005090].

[2] J. Polchinski, Dirichlet-branes and Ramond-Ramond charges, Phys. Rev. Lett. 75 (1995) 4724, [arXiv:hep-th/9510017].

[3] R. Minasian and G. Moore, K-theory and Ramond-Ramond charge, J. High Energy Phys. 11 (1997) 002, [arXiv:hep-th/9710230].

[4] E. Witten, D-Branes and K-Theory, J. High Energy Phys. 12 (1998) 019, [arXiv:hep-th/9810188]. 
[5] P. Hořava, Type IIA D-branes, K-theory, and matrix theory, Adv. Theor. Math. Phys. 2 (1999) 1373, [arXiv:hep-th/9812135].

[6] G. Moore and E. Witten, Self duality, Ramond-Ramond fields, and Ktheory, J. High Energy Phys. 05 (2000) 032, [arXiv:hep-th/9912279].

[7] D. S. Freed and M. J. Hopkins, On Ramond-Ramond fields and Ktheory, J. High Energy Phys. 05 (2000) 044, [arXiv:hep-th/0002027].

[8] P. Donovan and M. Karoubi, Graded Brauer groups and K-theory with local coefficients, Inst. Hautes Études Sci. Publ. Math. 38 (1970) 5.

[9] J. Rosenberg, Continuous trace $C^{*}$-algebras from the bundle theoretic point of view, J. Aust. Math. Soc. A47 (1989) 368.

[10] D. S. Freed and E. Witten, Anomalies in string theory with D-Branes, Asian J. Math. 3 (1999) 819, [arXiv:hep-th/9907189].

[11] A. Kapustin, D-branes in a topologically nontrivial B-field, Adv. Theor. Math. Phys. 4 (2000) 127, [arXiv:hep-th/9909089].

[12] P. Bouwknegt and V. Mathai, D-branes, B-fields and twisted K-theory, J. High Energy Phys. 03 (2000) 007, [arXiv: hep-th/0002023].

[13] S. Gukov, K-theory, reality, and orientifolds, Commun. Math. Phys. 210 (2000) 621, [arXiv:hep-th/9901042].

[14] K. Hori, D-branes, T-duality, and index theory, Adv. Theor. Math. Phys. 3 (1999) 281, [arXiv:hep-th/9902102].

[15] K. Olsen and R. Szabo, Constructing D-branes from K-Theory, Adv. Theor. Math. Phys. 3 (1999) 889, [arXiv:hep-th/9907140].

[16] E. Witten, String theory dynamics in various dimensions, Nucl. Phys. B443 (1995) 85, [arXiv:hep-th/9503124].

[17] P. K. Townsend, Four lectures on M-theory, in Trieste summer school in High Energy Physics and Cosmology, 1996, [arXiv:hep-th/9612121].

[18] M. J. Duff, M-theory (the theory formerly known as strings), Int. J. Mod. Phys. A11 (1996) 5623, [arXiv: hep-th/9608117].

[19] E. Witten, On flux quantization in M-theory and the effective action, J. Geom. Phys. 22 (1997) 1, [arXiv:hep-th/9609122]. 
[20] E. Witten, Duality relations among topological effects in string theory, J. High Energy Phys. 0005 (2000) 031, [arXiv:hep-th/9912086].

[21] M.J. Hopkins, I.M. Singer, Quadratic functions in geometry, topology, and M-theory, [arXiv:math.AT/0211216].

[22] E. Diaconescu, D. Freed and G. Moore, The M-theory 3-form and E8 gauge theory, [arXiv:hep-th/0312069].

[23] G. Moore and N. Saulina, T-duality, and the K-theoretic partition function of Type IIA superstring theory, Nucl. Phys. B670 (2003) 27, [arXiv:hep-th/0206092].

[24] V. Mathai and H. Sati, Some relations between twisted K-theory and $E_{8}$ gauge theory, J. High Energy Phys. 03 (2004) 016, [arXiv:hep-th/0312033].

[25] M. R. Douglas, D-branes, categories and $N=1$ supersymmetry, J. Math. Phys. 42 (2001) 2818. [arXiv:hep-th/0011017].

[26] A. Caldararu, S. Katz and E. Sharpe, D-branes, $B$ fields, and Ext groups, Adv. Theor. Math. Phys. 7 (2003) 381, [arXiv:hep-th/0302099].

[27] P. Aspinwall and A. Lawrence, Derived categories and zero-brane stability, J. High Energy Phys. 0108 (2001) 004, [arXiv:hep-th/0104147].

[28] I Brunner and J. Distler, Torsion D-branes in nongeometrical phases, Adv. Theor. Math. Phys. 5 (2002) 265, [arXiv:hep-th/0102018].

[29] J. Lott, R/Z index theory, Comm. Anal. Geom. 2 (1994), no. 2, 279.

[30] T. Killingback, Global anomalies, string theory and spacetime topology, Class. Quant. Grav. 5 (1988), 1169.

[31] S. Stolz and P. Teichner, What is an elliptic object?, to appear in the Proceedings of Graeme Segal's Birthday Conference, Oxford University Press, www.math.ucsd.edu/teichner/Preprints/Oxford.pdf .

[32] E. H. Brown and F. P. Peterson, A spectrum whose $\mathbb{Z} / p$ cohomology is the algebra of reduced p-th powers, Topology 5 (1966) 149.

[33] J. Milnor, The Steenrod algebra and its dual, Ann. of Math. (2) 67 (1958) 150. 
[34] D. Ravenel, Complex cobordism and stable homotopy groups of spheres, Academic Press, Inc., Orlando, FL, 1986.

[35] E. Brown, Jr. and F. Peterson, Relations among characteristic classes. I, Topology 3 (1964) suppl. 1, 39.

[36] M. J. Hopkins, H. R. Miller, M. Mahowald and P. Goerss, in preparation.

[37] M. Ando, H. J. Hopkins and N. P. Strickland, Elliptic spectra, the Witten genus and the theorem of the cube, Invent. math. 146 (2001) 595.

[38] J. Lubin and J. Tate, Formal moduli for one-parameter formal Lie groups, Bull. Soc. Math. France 94 (1966) 49.

[39] P. Hu and I. Kriz, Real-oriented homotopy theory and an analogue of the Adams-Novikov spectral sequence, Topology 40 (2001) 317.

[40] M. Ando, M. J. Hopkins, C. Rezk, Orientability with respect to tmf, in preparation.

[41] C. Rezk, The units in a ring spectrum and the logarithm, preprint, 2004.

[42] N. Kuhn, Morava K-theories and infinite loop spaces, Algebraic topology (Arcata, CA, 1986), Lecture Notes in Mathematics, Vol. 1370, Springer Verlag, Berlin, 1989, pp. 243-257.

[43] M. Ando, Isogenies of formal group laws and power operations in the cohomology theories $E_{n}$, Duke Math. J. 79 (1995), 423.

[44] J. P. May, $E_{\infty}$-ring spaces and $E_{\infty}$-ring spectra, with contributions by F. Quinn, N. Ray and J. Torenhave, Lecture Notes in Mathematics, Vol. 577, Springer Verlag, Berlin, 1977.

[45] G. Segal, Elliptic cohomology, Séminaire Bourbaki, Vol. 1987/88, Astérisque 161-162 (1988), Exp. No. 695 (1989) 187.

[46] J. Morava, Forms of K-theory, Math. Z. 201 (1989) 401.

[47] N. Baas, B. A. Dundas and J. Rognes, Two-vector bundles and forms of elliptic cohomology, to appear in the Proceedings of Graeme Segal's Birthday Conference, Oxford University Press, [arXiv:math.AT/0306027].

[48] I. Kriz and H. Sati, work in progress. 
[49] C. L. Douglas, On the twisted K-homology of simple Lie groups, [arXiv:math. AT/0402082].

[50] P. Hu, I. Kriz, Closed and open conformal field theories and their anomalies, to appear in Commun. Math. Phys., [arXiv:hep-th/0401061].

[51] K. Lechner, P. A. Marchetti and M. Tonin, Anomaly free effective action for the elementary M5-brane, Phys. Lett. B524 (2002) 199, [arXiv:hep-th/0107061].

[52] J. Gauntlett, Intersecting branes, lectures at APCTP winter school on Dualities of Gauge and String Theories, Sokcho, Korea, 1997, [arXiv:hep-th/9705011].

[53] R. Gopakumar, S. Minwalla, N. Seiberg and A. Strominger, OM theory in diverse dimensions, J. High energy Phys. 08 (2000) 008, [arXiv:hep-th/0006062].

[54] P. Hu and I. Kriz, Conformal field theory and elliptic cohomology, to appear in Advances in Mathematics.

[55] A. Sen, T-duality of p-branes, Mod. Phys. Lett. A11 (1996) 827, [arXiv:hep-th/9512203].

[56] O. Ganor, S. Ramgoolam and W. Taylor IV, Branes, fluxes and duality in M(atrix)-theory, Nucl. Phys. B492 (1997) 191, [arXiv:hep-th/9611202].

[57] Y. Rudyak, On Thom spectra, orientability, and cobordism, SpringerVerlag, Berlin, 1998.

[58] A. K. Bousfield, The localization of spectra with respect to homology, Topology 19 (1979), 257-281

[59] M. J. Hopkins and M. Mahowald, From elliptic curves to homotopy theory, preprint, 1998, http://hopf.math.purdue.edu/cgibin/generate?/Hopkins-Mahowald/eo2homotopy . 